\title{
芳基重氮盐参与的偶联反应研究进展
}

\author{
宣赏赐王晓丹王建伟赵保丽程凯* 齐陈泽
}

(绍兴文理学院 浙江省精细化学品传统工艺替代技术研究重点实验室 绍兴 312000)

\begin{abstract}
摘要 芳基重氮盐由于其价廉易得、反应活性高等特点作为芳基化试剂广泛应用于偶联反应中，近年来在有机合成中 越来越受到化学家们的重视. 综述了 2006 年以来的芳基重氮盐参与的偶联反应的研究情况，结合本课题组的工作，重 点探讨芳基重氮盐在新的碳碳键构筑方式、天然产物骨架的构建、非均相催化领域的延伸以及绿色合成等方面的新应 用和新进展.
\end{abstract}

关键词 芳基重氮盐; 偶联反应; 碳碳键的构筑; 绿色合成

\section{Research Progress on Cross-Coupling with Aryl Diazonium Salts}

\author{
Xuan, Shangci \\ Wang, Xiaodan \\ Wang, Jianwei \\ Zhao, Baoli \\ Cheng, Kai* \\ Qi, Chenze \\ (Zhejiang Key Laboratory of Alternative Technologies for Fine Chemicals Process, Shaoxing University, Shaoxing 312000)
}

\begin{abstract}
Aryl diazonium salts have been widely used in cross-coupling reaction due to its easily available and highly reactivity as arylation reagents, which have drawn more and more attention by chemists in organic synthesis in recent years. The research progress since 2006 on cross-coupling with aryl diazonium salts combined with our work is reviewed, focusing on new applications and new progress of aryl diazonium salts in new method to build carbon-carbon bond, with construction of the natural product skeleton, in the field of heterogeneous catalysis and other aspects of green synthesis.
\end{abstract}

Keywords aryl diazonium salts; cross-coupling; construction of $\mathrm{C}-\mathrm{C}$ bond; green synthesis

在钯催化的偶联反应中, 卤代烃是一类最常见的亲 电试剂，该类型反应能否顺利进行的关键环节是过渡金 属插入碳卤键从而来启动催化循环. 溴代烃、碘代烃虽 然反应活性相对较高但是制备成本高, 而较便宜且易得 的氯代烃反应活性很低, 难以发生金属插入启动反应, 而且卤代烃在反应进行的过程中生成大量废物卤化物 盐, 易对环境造成较大的危害. 而用磺酸苯酯类的亲电 试剂来代替卤代烃, 也仍然存在着一些不足, 如 $\mathrm{C}-\mathrm{O}$ 键难以断裂而导致的反应活性较低, 使得反应往往需要 较高温度, 由于大体积的离去基团增加后处理的难度 等, 限制了其进一步的应用. 近年来, 经由 $\mathrm{C}-\mathrm{N}$ 键活 化而不是 $\mathrm{C}-\mathrm{X}$ 键或 $\mathrm{C}-\mathrm{O}$ 键断裂启动金属催化循环来 构筑化合物的方法已经成为有机金属化学研究的新途 径和新思路. 在这类研究中, 芳基重氮盐因其独特的性
质成为偶联反应中极具研究价值的亲电试剂, 成为新的 研究热点. 其主要原因在于芳基重氮盐具有以下特点:

(1)芳基重氮盐可以很容易地由廉价的芳香胺合成, 而且收率很高, 几乎可以定量得到, 易于大量制备且便 于操作.

(2)在钯催化的偶联反应中, 芳基重氮盐作为亲电 试剂的反应活性高于其对应的卤代烃和芳香酯. 在反应 中随着氮气分子的离去形成芳香钯中间体来启动催化 循环, 该步骤通过热力学计算可知其所需要能量很低, 室温下就能进行，一般不会成为反应的决速步.

(3)以重氮盐为亲电试剂的偶联反应条件都较温和, 一般在室温下就能进行, 且通常不需要加入碱或配体等 作为添加剂, 易于后处理和分离.

(4)重氮基团在反应中以氮气分子的形式离去, 无

\footnotetext{
* E-mail: chengkai@usx.edu.cn

Received April 2, 2014; revised April 24, 2014; published online May 7, 2014.

Project supported by the National Natural Science Foundation of China (No. 21402123), the Zhejiang Provincial Natural Science Foundation of China (No. LQ14B020001), the Science Technology Department of Zhejiang Province (No. 2012R10014-15), the Education Department of Zhejiang Province (No. Y201328443) and the Science Technology Project of Shaoxing City (No. 2013014017).

国家自然科学基金(No. 21402123)、浙江省自然科学基金(No. LQ14B020001)、浙江省科技厅(No. 2012R10014-15)、浙江省教育厅(No. Y201328443) 和绍兴市科技计划(No. 2013014017)资助项目.
} 
残留, 符合绿色化学的要求. 反应一般不需要惰性气体 的保护，在空气中就能很好的进行.

(5)重氮基团相对于卤素在偶联反应的化学选择性 上也是具有优势的. 利用其与卤素取代基的活性差异可 以制备氯、溴或碘取代的产物, 具有良好的官能团耐受 性, 还可以分步制备非对称的并具备多取代结构的药物 和农药中间体.

由此可见, 芳基重氮盐参与的偶联反应的研究具有 十分重要的意义. 目前相关的各种报道涵盖了各种碳碳 键形成的方式: 芳-芳键(Suzuki-Miyaura 偶联, Stille 偶 联, Hiyama 偶联和自身偶联)、芳一烯键(Heck-Matsuda 偶联和氢化芳化反应)、芳一炔键(Sonogashira 偶联)以及 芳-羰键(芳烃的羰基化反应)的形成(Scheme 1).

芳基重氮盐在 $\mathrm{Pd}$ 催化反应中的新用途是目前研究 的重点, 芳基重氮盐参与的新型偶联反应将会广泛应用 于科研和工业. 该类反应的发展和应用不仅仅是对现有 领域的拓展, 更重要的是将开发新的领域并对那些几乎 空白的领域研究作出贡献. 而 Felpin ${ }^{[1]}$ 和李彦威等 ${ }^{[2]}$ 则 综述了芳基重氮盐的 Suzuki 偶联反应, Correia 等 ${ }^{[3]}$ 则着 眼在 Heck-Matsuda 反应, 今年, 北京大学王剑波教授分 别就叠氮化合物在偶联反应中的应用 ${ }^{[4]}$ 和芳基重氮盐在 有机合成中构筑 $\mathrm{C}-\mathrm{C} 、 \mathrm{C}-\mathrm{B}$ 和 $\mathrm{C}-\mathrm{S}$ 键的方面 ${ }^{[5]}$ 进行 了详尽的综述, 本文则结合本课题组的工作针对近几年 来芳基重氮盐参与的偶联反应的最新进展进行综述, 重 点是芳基重氮盐在新的碳碳键构建方式, 非均相催化领 域的延伸、天然产物骨架的构建和绿色合成等方面的最 新进展.

\section{1 新的碳碳键构建方式}

近年来，随着对芳基重氮盐反应机制研究的深入， 在传统的芳基重氮盐参与的偶联反应基础上 ${ }^{[6]}$, 一些新 的碳碳键的构筑方式不断被报道. 本文第一部分着重于 新的碳碳键构筑方式的综述.

\subsection{Hiyama 交叉偶联反应}

有机硅试剂由于其廉价、易得、对环境更友好、反 应稳定性好等优点, 常作为高毒性的锡试剂的替代物应 用于联芳烃的合成中(Hiyama 偶联反应). 我们课题组 ${ }^{[7]}$
报道了一种钯催化芳基重氮盐参与的 Hiyama 偶联合成 联苯类化合物的新方法，室温下无需隔绝空气就可以高 产率的得到相应的联苯化合物, 而且该方法无需膦配体 和氟离子或氢氧根离子的加入来促进反应进行. 我们通 过热力学计算推测了芳基重氮盐参与的 Hiyama 偶联的 催化机理, 发现无氟存在下四氟硼酸根离子进攻硅醚中 的 $\mathrm{Si}$ 中心原子促进硅醚基团的离去，是促进反应进行 的关键步骤(Eq. 1).

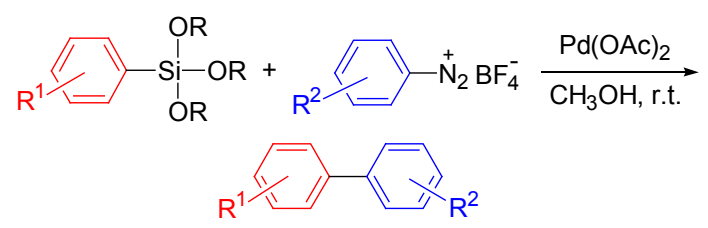

\subsection{Sonogashira 交叉偶联反应}

Cacchi 等 ${ }^{[8]}$ 首次报道了芳基重氮盐和端炔在 $\mathrm{Pd}-$ $\mathrm{Cu}(\mathrm{I})$ 共催化下，反应中加入四丁基碘化铵作添加剂，二 乙胺作碱，以 $71 \%$ ～91\%的偶联产率得到二苯乙炔类底 物，炔烃在反应过程中得到保持。该反应条件温和，通 过多米诺重氮碘代/Sonogashira 偶联历程, 实现了芳基 重氮盐的炔基化(Eq. 2). 该过程还可以通过芳香胺同苯 乙炔的一锅法多米诺偶联反应进行，作者认为 $\sigma$-碘代芳 钯中间体和原位生成的炔铜化合物之间的反应活性是 反应进行的关键. 炔丙醇、脂肪族端炔，炔基硅醚，杂环 炔烃都可以反应，芳环上氨基、羟基也不受影响.

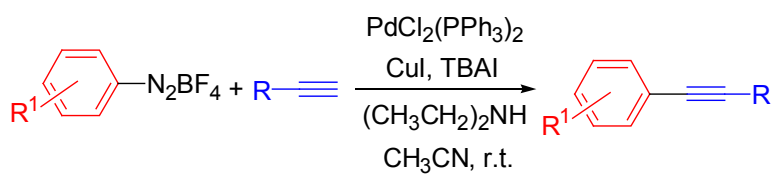

Beller 等 ${ }^{[9]}$ 报道了直接从苯胺出发, 通过与亚硝酸 叔丁酯和醋酸原位生成醋酸阴离子的芳基重氮盐, 在醋 酸钯催化下, 以 tri(2-furyl)phosphine (TFP)作为配体与 芳基乙炔发生 Sonogashira 偶联反应(Eq. 3). 该反应条件

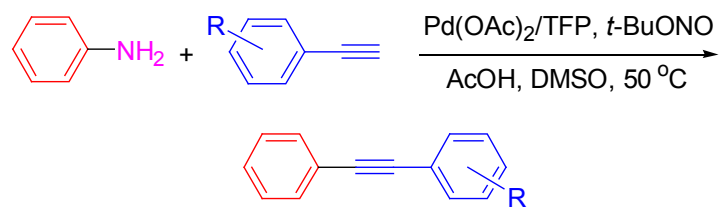

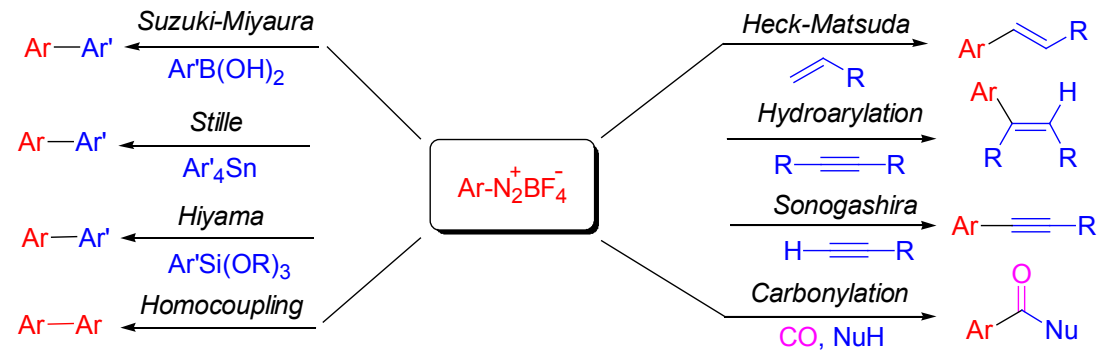

Scheme 1 
温和, 无需添加额外的碱, 炔丙胺、三甲硅基乙炔和脂 肪族的端炔也都可以顺利反应，产率 63\% 93\%不等. 作者认为体系中的醋酸根阴离子还起到了辅助脱除炔 烃质子的作用, 促进反应进行.

Sarkar 等 ${ }^{[10]}$ 报道了在 $\mathrm{Pd}-\mathrm{Au}(\mathrm{I})$ 共催化下的 Sonogashira 反应，以 2,6-二甲基吡啶作为碱，室温下即可高 产率得到相应交叉偶联炔烃产物, 以及少量炔烃自身偶 联产物(Eq. 4).

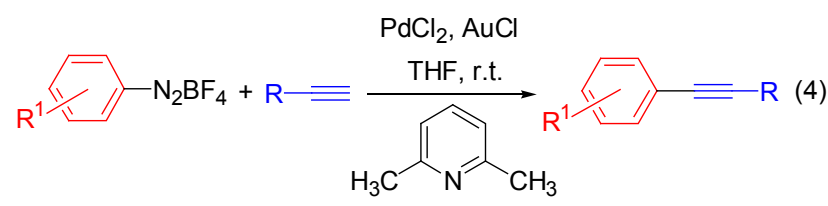

当邻位硝基取代时, 在 DBMP (2,6-ditert-butyl-4methylpyridine)辅助下可以发生金催化分子内串联环化 反应, 得到异噁唑衍生物, 提供了一种潜在的异噁唑单 元结构的构筑方法(Eq. 5).<smiles>[R]C#C[C@H](C)[C@@H](C)NC(C)C</smiles><smiles>[R]C(=O)c1onc2ccccc12</smiles>

$(29 \%)$<smiles>[R]C(=O)c1onc2ccc([N+](=O)[O-])cc12</smiles>

(33\%)

\section{3 插羰偶联反应}

Beller 等 ${ }^{[1]}$ 在之前报道的芳基重氮盐参与的 Sonogashira 偶联反应基础之上, 将该条件成功应用于一氧化 碳插羰反应. 他们报道了通过苯胺与亚硝酸叔丁酯、醋 酸形成的叔丁氧基重氮盐为关键步骤, 在 $\operatorname{Pd}(\mathrm{II})$ 催化下 $1.0 \mathrm{MPa}$ 压力的 $\mathrm{CO}$ 中合成了 $N$-苯基苯甲酰胺(Eq. 6). 作 者推测其作用模式与 Sonogashira 反应相似, 通过醋酸 根离子辅助脱除苯胺质子来促进反应进行.

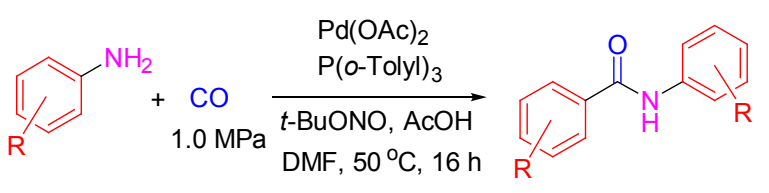

Beller 等 ${ }^{[12]}$ 改进了反应体系, 只需要在 $100 \mathrm{kPa} \mathrm{CO}$ 压力下就可以以苯胺来合成炔基化的苯甲酮类化合物, 该反应通过混合溶剂的引入, 将反应温度降低至接近室 温，产率 55\% 93\%. 该体系还同样适用于脂肪族炔烃， 产率 68\% 92\%. 反应中很好的抑制了苯胺自身生成 $N-$
苯基苯甲酰胺的副反应(Eq. 7).

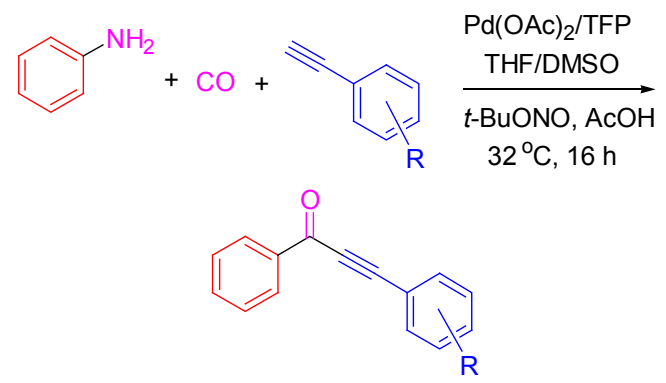

\section{4 氢化芳基化反应}

Cacchi 等 ${ }^{[13]}$ 在发现芳基重氮盐参与的 Sonogashira 偶联反应之前, 研究炔烃与芳基重氮盐的反应时发现, 当加入三苯基硅氢作为还原剂时，室温下就可以得到炔 烃氢化芳基化的产物. 反应可以高立体选择性的得到顺 式加成产物(Eq. 8). 当炔烃两端是非对称基团时, 生成 了芳基之间反式为主的产物. 该反应也可以通过一锅法 策略以苯胺作为初始原料实现(Eq. 9).<smiles>[R]C=C([R])c1cccc([R])c1</smiles>

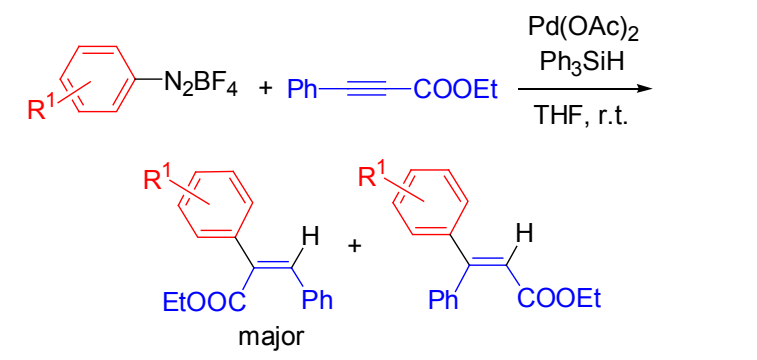

Cacchi 等 ${ }^{[14]}$ 还利用多米诺 Sonogashira 偶联反应的 特性, 零价 Pd 催化下加入四丁基碘化铵作添加剂以促 进重氮碘化，生成碘化芳钯中间体，通过与酰基保护的 邻氨基苯乙炔络合，经分子内关环反应合成 3-芳基吲哚 衍生物(Eq. 10), 形成了一条很巧妙的吲哚结构构筑的 思路. 作者通过条件篮选很好地抑制了原料自身直接关 环以及硒化芳钯中间体直接还原消除所得到的副产物.

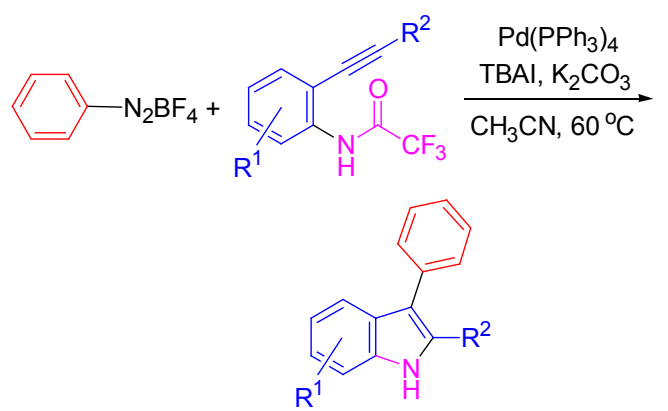




\section{5 自身偶联反应}

卤代芳烃自身偶联是合成对称性联芳烃最有效的 方法，而传统的 Ullmann 反应需要高温下和高用量的催 化剂而大大增加了应用成本. 利用芳基重氮盐直接自身 偶联则可以以便宜易得的苯胺为初始原料, 通常在室温 下即可进行.

Hanna Jr 等 ${ }^{[15]}$ 报道了钯催化的芳基重氮盐的自身偶 联反应，甲醇回流下可以得到自身偶联产物、还原脱氮 反应和甲醇亲核取代产物(Eq. 11)。

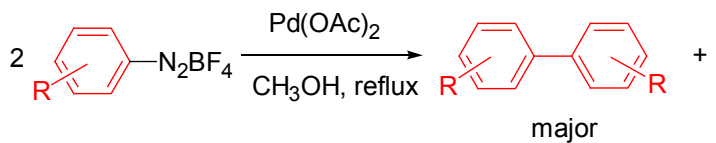

Litvic 等 ${ }^{[16]}$ 报道了铜催化的芳基重氮盐的自身偶联 反应. 当芳基重氮盐连有供电子基团时，使用催化量的 $\mathrm{Cu}(\mathrm{II})(\mathrm{OTf})_{2}$ 以 2,2-联吡啶作为配体, 铜粉作为终点还原 剂即可以使反应进行. 而当芳基重氮盐上连有吸电子基 才时, 则要用化学计量的 $\mathrm{Cu}(\mathrm{I}) \mathrm{OTf}$ 作促进剂, 才能得到 偶联产物. 反应中还同时检测到偶氮苯副产物(Eq. 12).

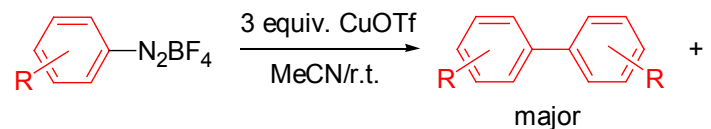<smiles>[R]c1ccc(N=Nc2cccc(N=Nc3ccccc3)c2)cc1</smiles>

我们课题组 ${ }^{[17]}$ 在研究中发现, 二价铁可以促进重氮 盐的分解和苯基自由基的生成. 我们发展了一种化学计 量的铁促进的芳基重氮盐的自身偶联反应. 该反应通过 单电子转移的反应机理进行, 反应条件温和且无需添加 剂, 具有良好的官能团耐受性, 可以高产率地得到对称 的联苯类化合物(Eq. 13).

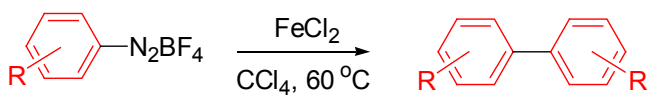

而且, 我们在进一步研究中发现 ${ }^{[18]}$, 上述反应中生 成的 $\mathrm{Fe}^{3+}$ 可以通过选择合适的还原剂重新还原为 $\mathrm{Fe}^{2+}$, 从而完成催化循环，使得反应可以在催化量的 $\mathrm{Fe}^{2+}$ 作用 下进行，提供了一条制备对称的联苯的经济高效的方法 (Eq. 14).

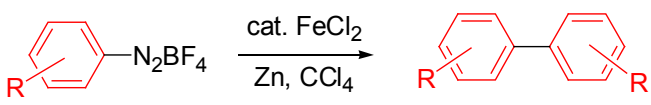

Chi 等 ${ }^{[19]}$ 将芳基重氮盐的自身偶联应用于连四苯的
合成中，通过实验证明了芳基重氮盐的活性高于卤代芳 烃. 在醋酸钯的催化下能高效地构筑联芳烃，为多芳烃 化合物的合成提供了新的选择(Scheme 2).

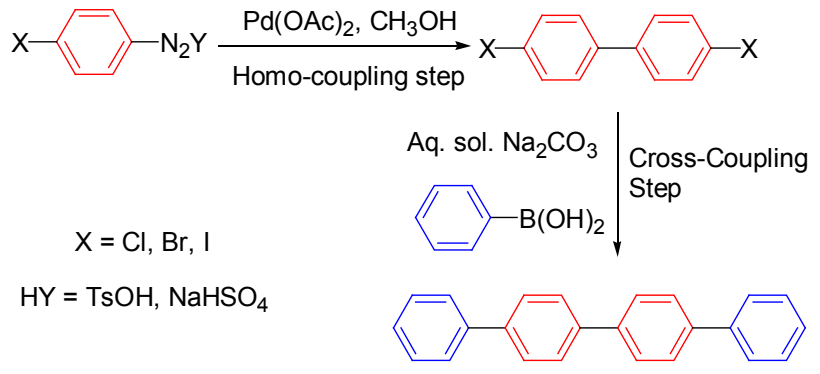

\section{Scheme 2}

\section{6 其它的碳碳键形成反应}

苏为科等 ${ }^{[20]}$ 报道了芳基重氮盐与过量的多氟代苯 在 $\mathrm{CuI}$ 催化下通过 $\mathrm{C}-\mathrm{H}$ 键的断裂合成多氟联苯产物. 反应具有较好的官能团相容性, 条件温和、反应时间短 且无需昂贵的配体, 即可取得较好的结果, 反应产率 25\% 89\%不等(Eq. 15). 该方法可以无需预官能化就可 以完成缺电子的多氟代苯的直接芳基化. 反应中通过添 加四丁基碘化铵，发生重氮碘化促进碘代芳铜中间体的 形成, 利用碱脱除 $\mathrm{HI}$ 使反应顺利进行, 无碘存在下只会 发生芳基重氮盐自身偶联反应. 同时也可以通过一锅法 以苯胺为底物一步制得偶联产物, 避免了四氟硼酸重氮 盐的分离, 反应产率较好(Eq. 16).
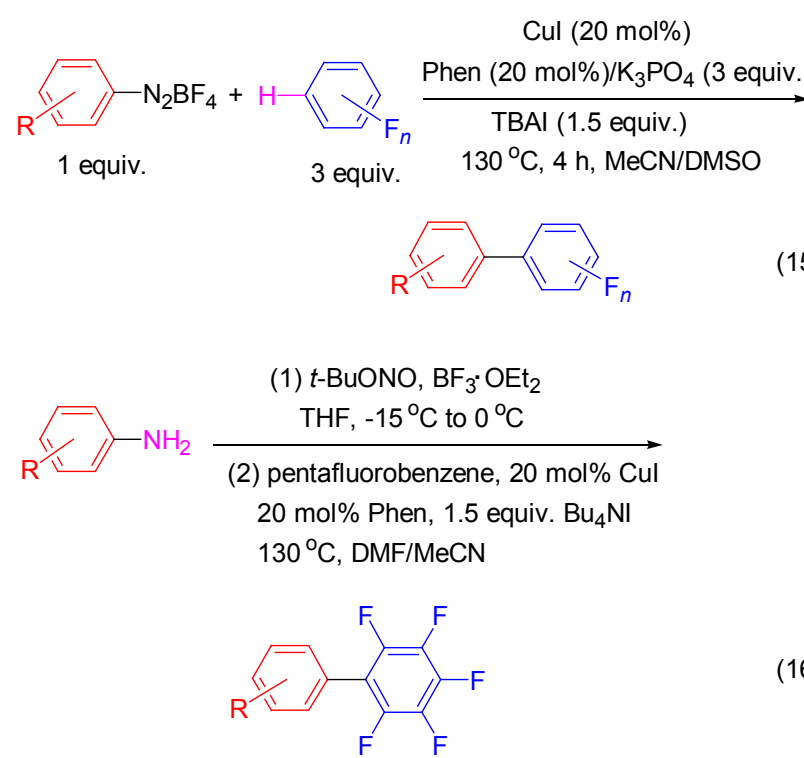

朱强研究员等 ${ }^{[21]}$ 发现了一种无需过渡金属催化的 通过芳基重氮盐与异腈化合物在零度下反应，仅需 20 min 就可以高选择性地得到苯甲酰胺类化合物. 反应溶 剂中的水参与到反应中. 反应在碱或者丙酮作用下发生 单电子转移(SET)分解成自由基，与异腈发生加成生成 
氮杂炔正离子，水合得到苯甲酰胺(Eq. 17).

$$
\text { }+\mathrm{R}^{2}-\mathrm{N} \equiv \mathrm{C} \frac{\mathrm{N}_{2} \mathrm{BF}_{4} \mathrm{C}, 20 \mathrm{~min}}{\mathrm{Cs}_{3} \mathrm{CO}_{3} \text { (1.1 equiv.) }}
$$

Sigman 等 ${ }^{[22]}$ 发展了一种新的碳碳键的形成方式, 芳基重氮盐与烯烃和芳基硼酸同时存在下发生三组分 反应，高选择性地得到二芳基烷烃(Eq. 18).

$$
\text { 等 }
$$

在反应条件的优化中作者发现，三组分反应产物、 Heck 类型产物、Suzuki 类型产物都可以检测到. 当换用 THF 或者 DMA 作溶剂时, 发生的是 Heck 类型的反应; 而用叔丁醇作溶剂则是以三组分反应为主(Eq. 19). 作 者基于此提出的机理也是以 Heck 反应途径先发生, 钯 重新插入的中间体被苯硣酸捕捉形成三组分反应.

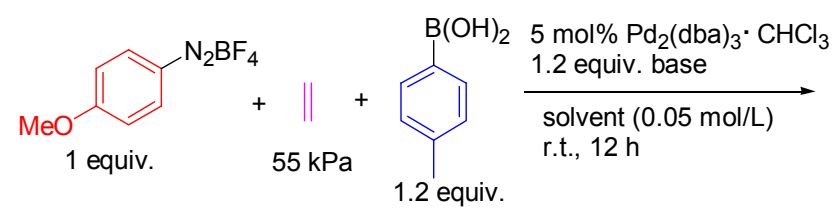<smiles>C=CC1=CC=C(OC)C[C+]1C=C(C)C=C1C=CC(C)=CC1</smiles>

Antunes 等 ${ }^{[23]}$ 报道了芳基重氮盐在有氧的条件下, $\mathrm{Pd}$ 催化下发生 Baylis-Hillman 加合反应得到 $\alpha$-苠基- $\beta$ 酮酯. 该合成方法类似于芳基重氮盐参与的 Heck 反应, 但由于底物加成后的含钯中间体的 $\beta$-消除方向不同而 生成了不同的产物(Eq. 20).

Correia 等 ${ }^{[24]}$ 报道了一种钯催化的吲哚、苯并呋喃和 苯并噻吩的 $\mathrm{C}-\mathrm{H}$ 芳基化反应. 有意思的是, 吲哚、苯 并呋喃芳基化的位置是 C-2 位, 而苯并噻吩则发生在 C-3 位上. 这可能是由于形成的芳钯正离子中间体的稳 定性所决定的(Scheme 3).<smiles>[R]C(O)C(=C)C(=O)OC</smiles>

$\mathrm{R}^{1}=\mathrm{CH}_{2} \mathrm{CH}_{3} ; \mathrm{R}^{1}=p-\mathrm{NO}_{2} \mathrm{C}_{6} \mathrm{H}_{4}$ or $\mathrm{R}^{1}=\mathrm{Hexyl}$
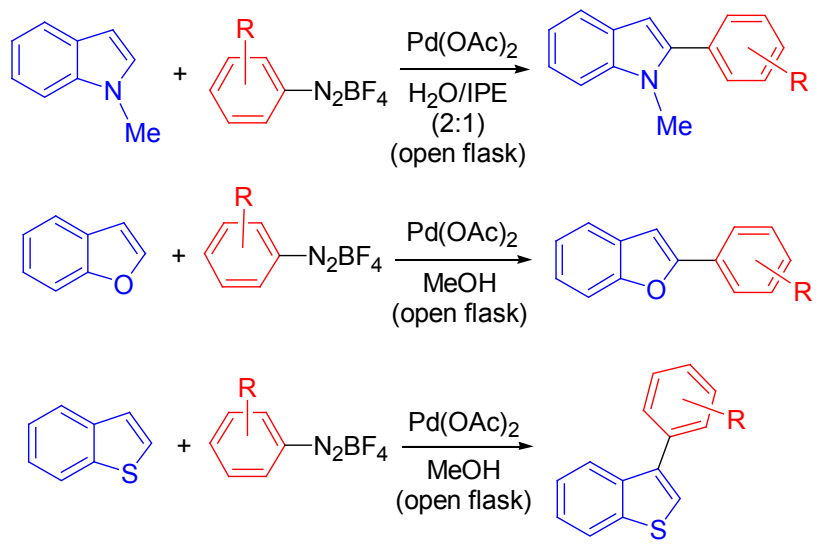

Scheme 3

\section{2 天然产物骨架的构建}

芳基重氮盐参与的 Heck 反应由于其良好的化学选 择性和极温和的反应条件使得其在天然产物的合成中 扮演了重要角色. Correia 课题组多年以来一直致力于芳 基重氮盐参与的 Heck-Matsuda 反应在天然产物合成方 面的应用. 早在 2003 年, Correia ${ }^{[25]}$ 就发展了一种室温下 环状烯胺通过芳基化短时间内即可构筑( $2 S, 5 R)$-苯基脯 氨酸甲酯结构, 可以来合成 Schramm's $C$-Azanucleoside (Eq. 21).
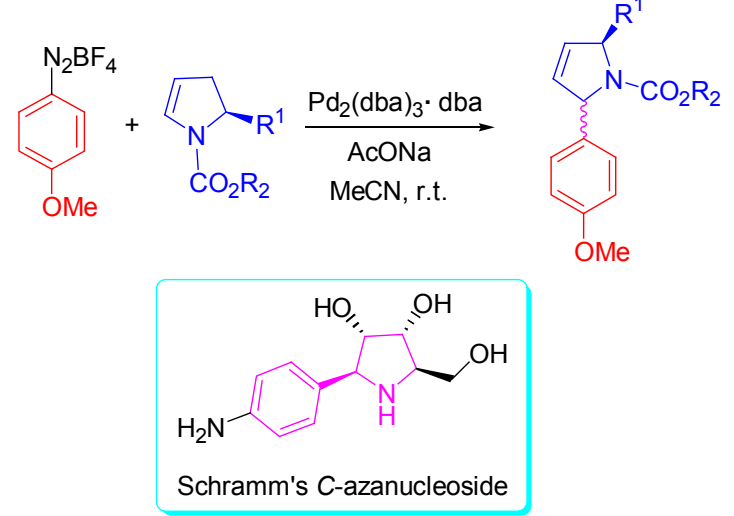

Correia 等 ${ }^{[26]}$ 报道了顺丁烯二酸酐的单或者双芳基 化来合成海洋类生物碱 Prepolycitrin A 和 Polycitrin A (Scheme 4).

Correia 等 ${ }^{[27]}$ 报道了立体选择性的手性二氢呋喃的 Heck-Matsuda 型芳基化, 并以此为关键步骤完成了(一)- 

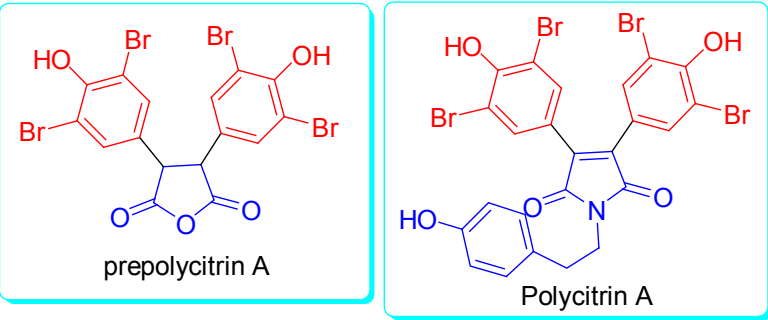

\section{Scheme 4}

Isoaltholactone 的全合成(Eq. 22).

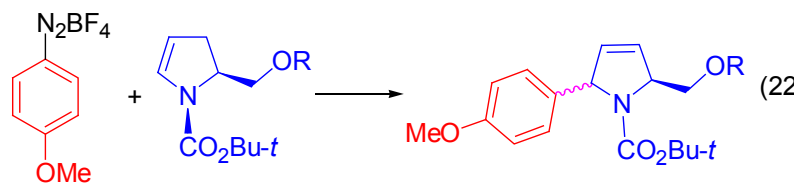

$$
\text { (-)-isoaltholactone }
$$

Correia 等 ${ }^{[28]}$ 利用 3-吡咯啉的芳基化构筑 4-芳基-2吡咯啉酮, 以合成克级的 $( \pm)$-Rolipram 等化合物 (Scheme 5).

Correia 等 ${ }^{[29]}$ 报道了环状的丙烯酸酯衍生物的芳基 化来合成抗抑郁药物 $( \pm$ )-Paroxetine (Scheme 6).

Correia 等 ${ }^{[30]}$ 发展了一种立体和区域选择性的, 以 芳基重氮盐同不饱和环胺的分子间的 Heck 反应作为关 键步骤, 完成了用作神经兴奋剂的芳基 Kainoids 的全合 成(Scheme 7).

Correia 等 $^{[31}$ 通过苯乙烯与芳基重氮盐的 Heck 反应 来简便高效立体选择性的合成白莍芦醇和 DMU-212 及 类似物(Eq. 23).
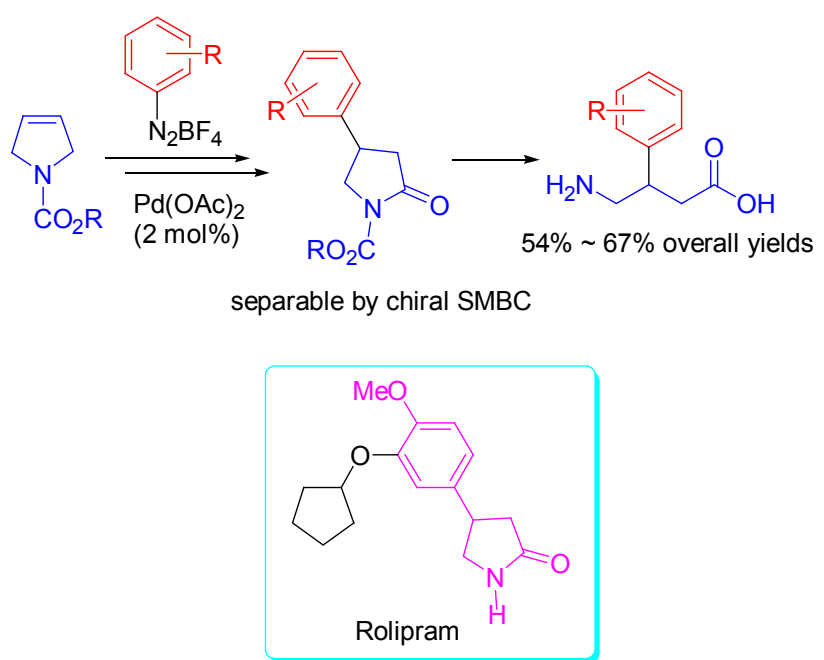

Scheme 5<smiles>COC(=O)C1=CCCN(C(C)=O)C1</smiles>

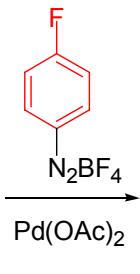

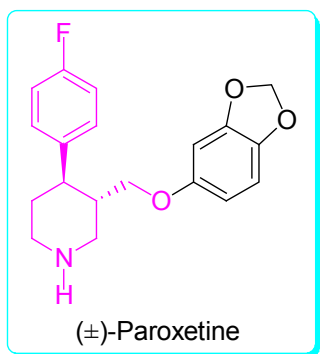

Scheme 6

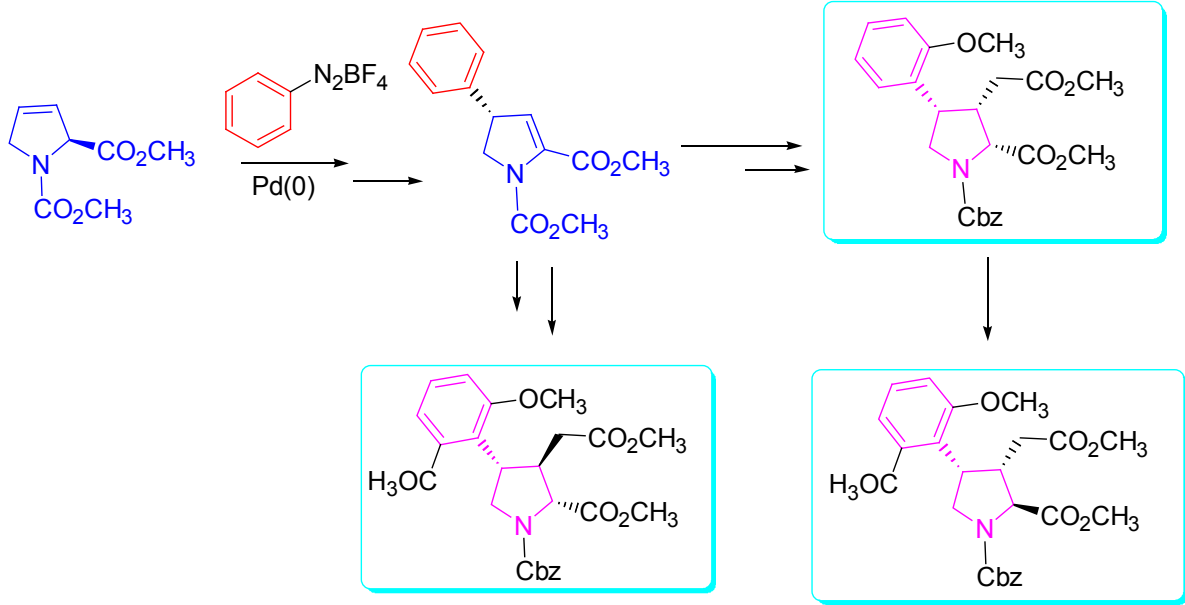

Scheme 7 
<smiles>C=CC1C=CC(OC(C)=O)=CC1</smiles>

$\mathrm{Pd}_{2}(\mathrm{dba})_{3} \cdot \mathrm{dba}$ $\mathrm{NaOAc}, \mathrm{PhCN}$ r.t., $1 \mathrm{~h}, 95 \%$

$\mathrm{NaOH}, \mathrm{THF}$ reflux, $2 \mathrm{~h}, 98 \%$<smiles>COc1cc(/C=C/c2ccc(O)cc2)cc(OC)c1OC</smiles>

Correia 等 ${ }^{[32]}$ 发展了 Isoaltholactone 和 Goniothalesdiol 的氮杂类似物的立体选择性合成(Eq. 24).
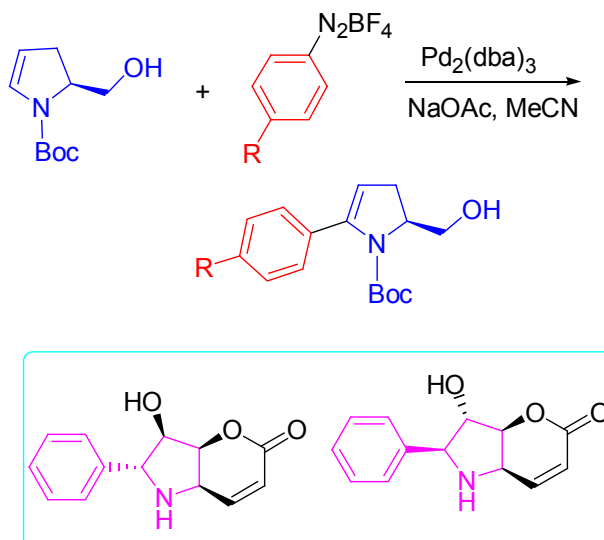

aza-isoaltholactone

aza-altholactone

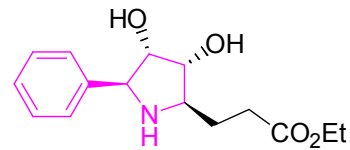

aza-analogue of goniothalesdiol

Correia 等 ${ }^{[33]}$ 利用底物导向的 Heck 与芳基重氮盐的
反应，完成了区域和立体选择性的烯丙胺衍生物的芳基 化并应用于 Naftifine 和 Abamines 的合成中(Scheme 8).

Correia 等 ${ }^{[34]}$ 报道了 2-杂原子取代的丙烯酸酯的合 成. 而 3,4-二氢异香豆素和苯酞也可以利用该策略, 通 过邻位引入羧基以发生分子内环化而形成内酯结构 ${ }^{[35]}$ (Scheme 9).

Correia 等 ${ }^{[36]}$ 发展了一种不对称 $\beta, \beta$-二芳基丙烯酸 酯的立体选择性合成，可以来构建 $\beta, \beta$-二芳基丙烯乙 酯、3-芳基吲哚和 4-芳基-3,4-二氢-2-喹啉铜结构单元以 及(一)-Indatraline 的全合成(Scheme 10).

Correia 等 ${ }^{[37]}$ 发展了一种取代的环戊烯的立体选择 性芳基化反应，通过底物导向的 Heck 反应可以用来鞘 氨醇 1-膦受体 (S1P1) 收缩剂 VPC01091 的全合成 (Scheme 11).

Correia 等 ${ }^{[38]}$ 报道了在钯催化下无保护邻羟基肉桂 酸酯同四氟嗍酸重氮盐的交叉偶联环化反应合成了 4芳基香豆素, 在有氧和无需额外配体的条件下, $60{ }^{\circ} \mathrm{C}$ 下 即可反应，产率 $41 \% \sim 91 \%$ 不等. 并且以此为关键步骤 的总产率 $30 \%$ 合成了 ee 值高达 $98 \%$ 的 $(R)$-Tolterodine (Scheme 12).

Correia 等 ${ }^{[39]}$ 发展了多样性全合成天然的抗症疾的 marinoquinoline 的 A、B、C、E 环和人工合成的类似物 (Scheme 13).

Correia 等 ${ }^{[40]}$ 报道了对映体选择性的手性双噁唑啉 辅助的 Matsuda-Heck 反应完成非活化的环状烯烃的芳 基化反应(Eq. 25).

Correia 等 $^{[41]}$ 通过 Heck 反应合成了五溴 pseudilin 及 其他芳基取代吡咯衍生物(Eq. 26).

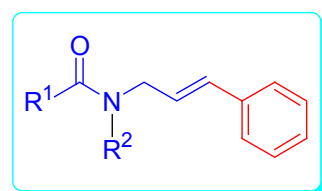

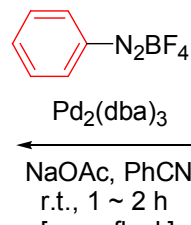<smiles>[R]C(=O)N([R])C/C=C\C</smiles>

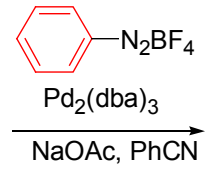

$80^{\circ} \mathrm{C}, 1 \mathrm{~h}$

[open-flask]

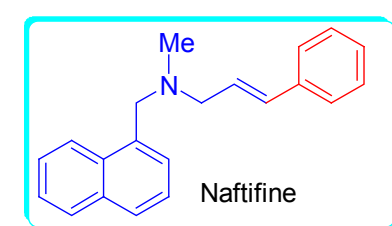

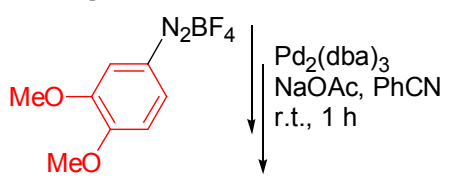

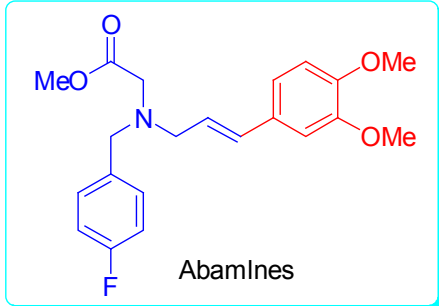

Scheme 8 
有机化学

综述与进展

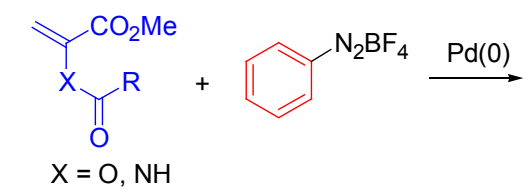<smiles>[R]C(=O)C(=Cc1ccccc1)C(C)=O</smiles>

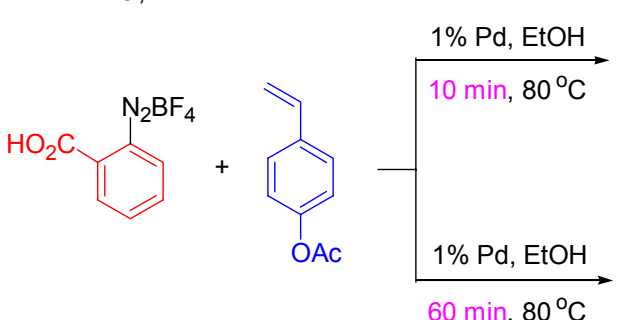<smiles>[R]c1ccc(/C=C/c2ccccc2C(=O)O)cc1</smiles>

$$
\mathrm{R}=\mathrm{OAc}(58 \%)+\mathrm{R}=\mathrm{OH}(18 \%)
$$
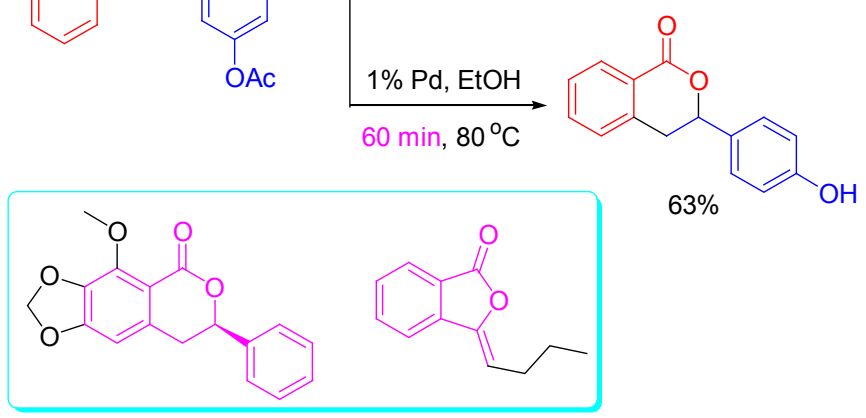

Scheme 9

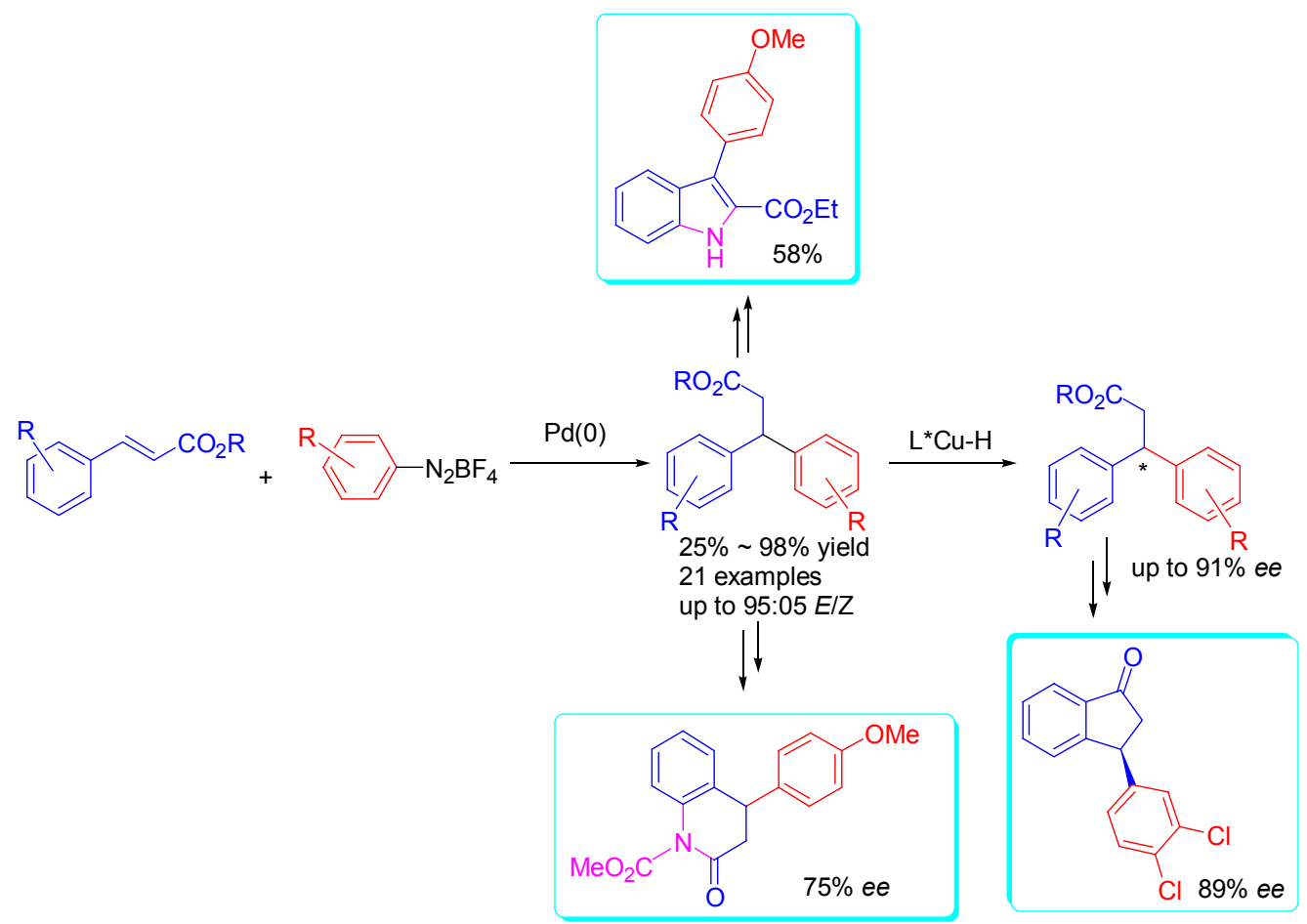

Scheme 10

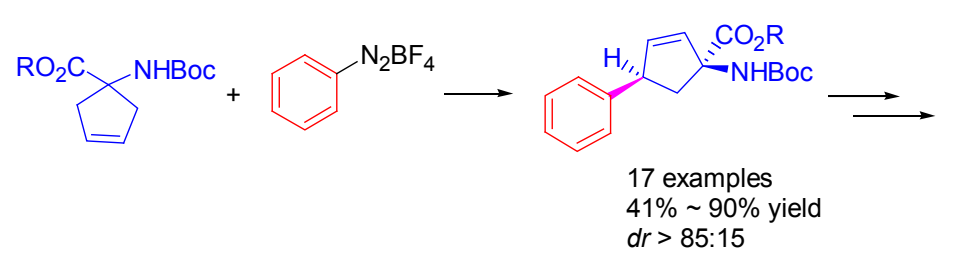

Scheme 11

1750

http://sioc-journal.cn/

(C) 2014 Chinese Chemical Society \& SIOC, CAS

Chin. J. Org. Chem. 2014, 34, 1743 1758 
<smiles></smiles>

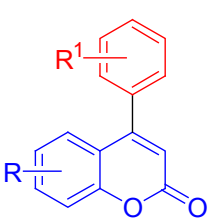

$41 \% \sim 91 \%$ yield 15 examples

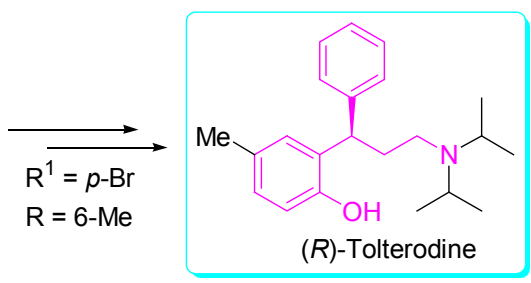

Scheme 12
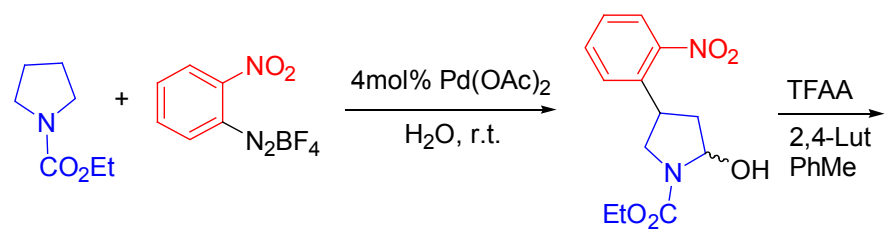

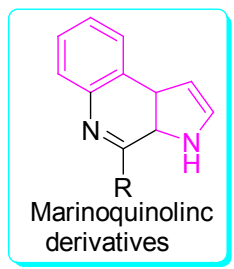

Scheme 13<smiles>CC(=O)C1(C(C)=O)CC=CC1</smiles>

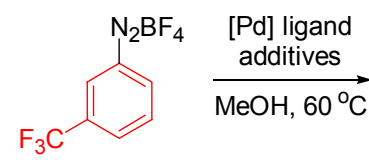<smiles>COC(=O)C1(C(C)=O)C=CC(c2cccc(C(F)(F)F)c2)C1</smiles><smiles>c1ccc(C2COC(c3cccc(C4=NC(c5ccccc5)CO4)n3)=N2)cc1</smiles><smiles>c1ccc(CC2COC(C3=NC(Cc4ccccc4)CO3)=N2)cc1</smiles><smiles>c1ccc2c(c1)C[C@H]1OC(CC3=N[C@@H]4Cc5ccccc5[C@H]4O3)=N[C@@H]21</smiles>

(26)

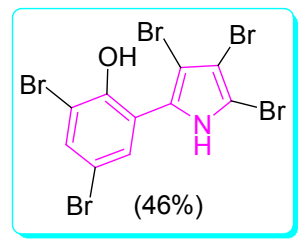

最近, Correia 等 ${ }^{[42]}$ 发展了一种分子间立体选择性的 烯丙醇与芳基重氮盐通过 Heck-Matsuda 反应构筑 $\beta$-Aryl- $\gamma$-lactone 和 $\beta$-芳基醛类化合物. 作者已经将该方 法应用在天然产物的全合成上，以 5 步 50\%的总产率得 到 Aryl-pyrrolidine (Scheme 14).

Correia 等 ${ }^{[43]}$ 发现芳基重氮盐和肉桂酸酯类化合物 的 Heck 反应具有良好的区域选择性, 并已经用于 (士)-Indatraline 和( \pm )-Sertraline 的全合成中(Eq. 27).

Cacchi 等 ${ }^{[44]}$ 报道了芳基重氮盐与 4-羟基-2-丁烯酸 甲酯合成了 4-芳基丁烯酸内酯, 在钯的催化下, 以甲醇 为溶剂, 反应条件温和, 且产率中等. 该方法可以通过

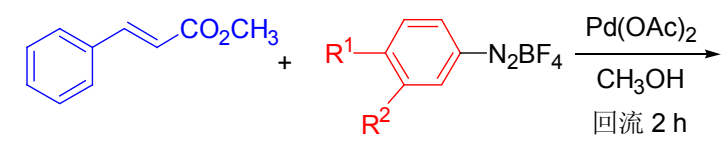<smiles>[R]c1ccc(C(=CC(C)=O)c2ccccc2)cc1[R]</smiles>

高效的两步过程无需分离丁烯酸内酯中间体即可构筑 Rubrolide 的骨架结构(Scheme 15).

Schmidt 等 ${ }^{[45]}$ 报道了有关对烷氧基取代的芳基重氮 盐参与的 Heck 反应, 通过加入醋酸钠, 可以大大促进其 转化的效率. 该反应不但可以用来合成 acetamidocinnamates. 还可以来合成 aripiprazole 的关键片段 7-羟基-3, 4-二氢喹啉酮(Scheme 16). 当将烯基同时引入到吸电子 的芳基和酯基时，则其发生 Heck 反应时就会产生区域 选择性的问题. 而 Felpin 等 ${ }^{[46]}$ 则系统地研究了 2-芳基丙 烯酸酯进行 Heck 反应的区域选择性，并通过密度泛函 理论(DFT)计算对实验结果予以证明. 其中该体系中钯 的用量可以降到 $1 \mathrm{~mol} \%$ (Eq. 28). 


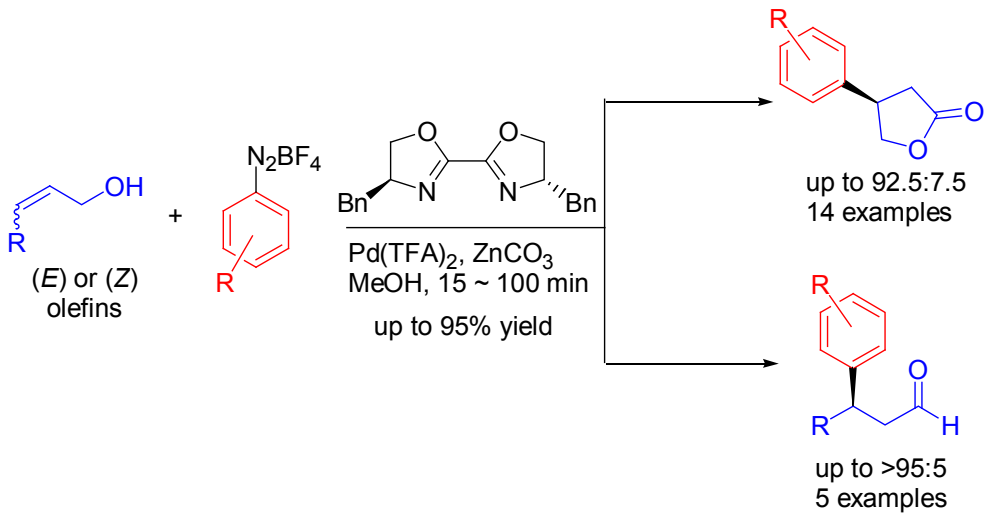

Scheme 14
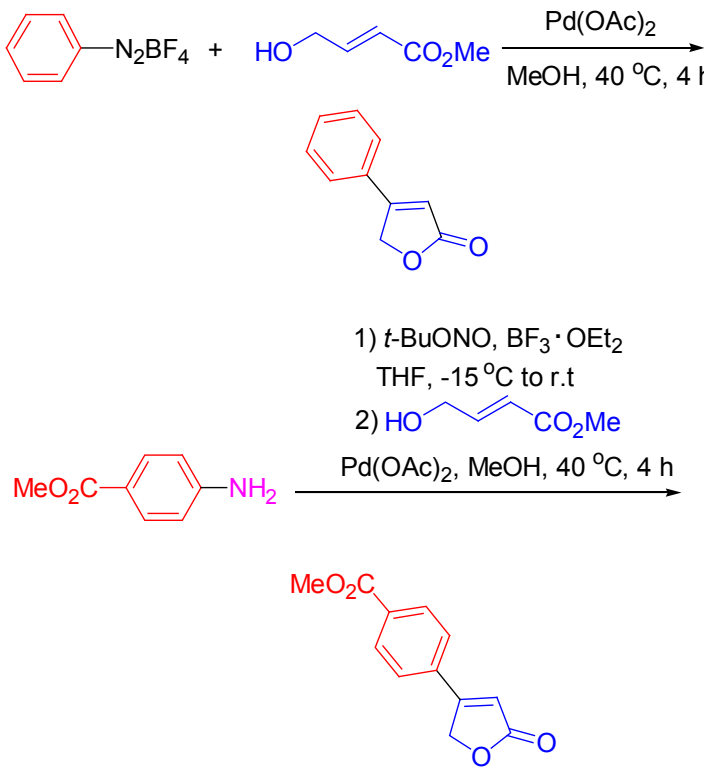

$73 \%$

Scheme 15

R $-\mathrm{N}_{2} \mathrm{BF}_{4}+\prod_{\mathrm{O}}^{\mathrm{O}} \mathrm{OCH}_{3} \frac{\mathrm{Pd}(\mathrm{OAc})_{2}}{\text { r.t. }}$<smiles>[R]c1ccc(/C=C/C(=O)OC)cc1</smiles><smiles>C=C(NC(=O)C(C)=O)C(=O)OC</smiles>

Scheme 16

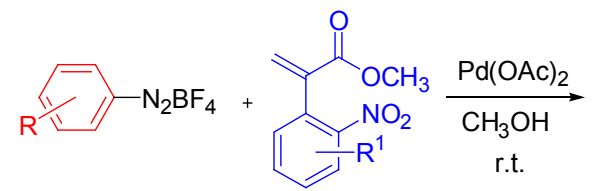<smiles>[R]c1ccc(/C=C(/C(=O)OC)c2ccccc2[R])cc1</smiles>

Felpin 等还首次通过钯催化分子内的 Heck 还原环 化反应 $(\mathrm{HRC})$ 分别合成了吲哚啉䣨 ${ }^{[47]}$ 和喹诺酮类 ${ }^{[48]}$ 化 合物，为该类型反应的发展又开辟出了一片新的天地 (Scheme 17).
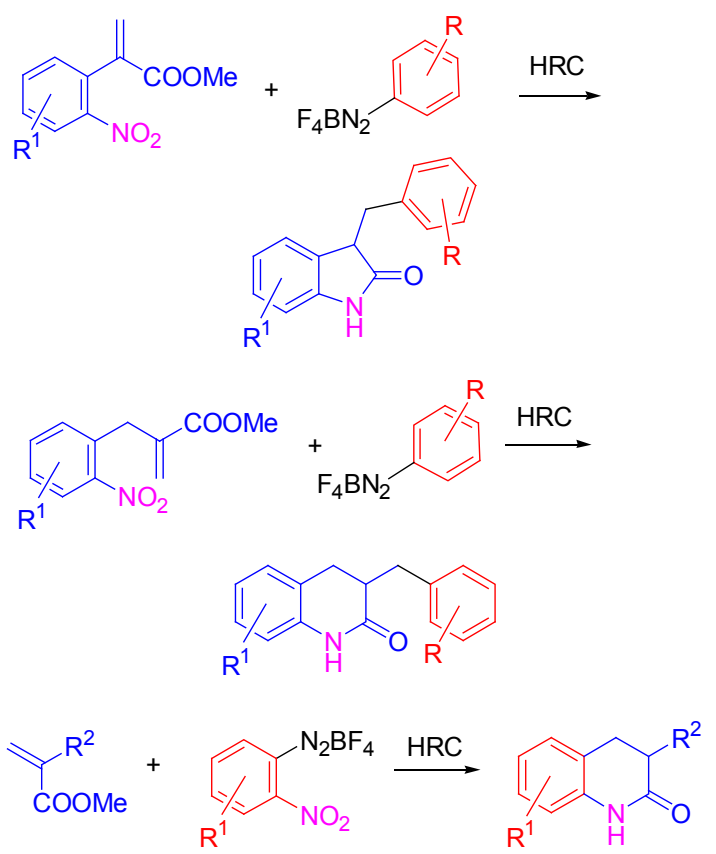

Scheme 17

Correia 等 ${ }^{[49]}$ 报道了烯醇醚类化合物也可以与芳基 重氮盐发生 Heck 反应，来合成黄酮类化合物(Eq. 29). 


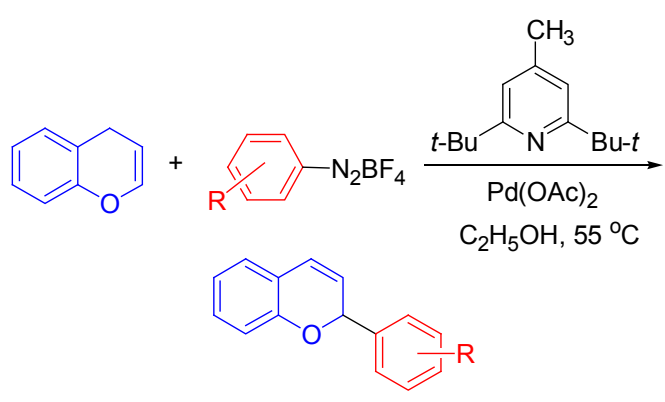

Correia 等 ${ }^{[50]}$ 报道了以芳基重氮盐同烯丙基醋酸酯 的 Heck 反应作为关键步骤, 合成具有环状内酯结构的 Kavalactones (Eq. 30).

$$
\begin{aligned}
& \text { 至 } \\
& \text { 邹 } R
\end{aligned}
$$

Correia 等 ${ }^{[51]}$ 报道了芳基重氮盐通过与 2-乙烯基(2氢)吡喃酮反应作为关键步骤, 直接或通过氧化还原制 备了多种 2-乙烯基吡喃酮 Kavalactones 芳基化衍生物, 并成功改变了反应条件促进了带吸电子基团的重氮盐 与 2-乙烯基(2-氢)吡喃酮的偶联反应(Eq. 31).

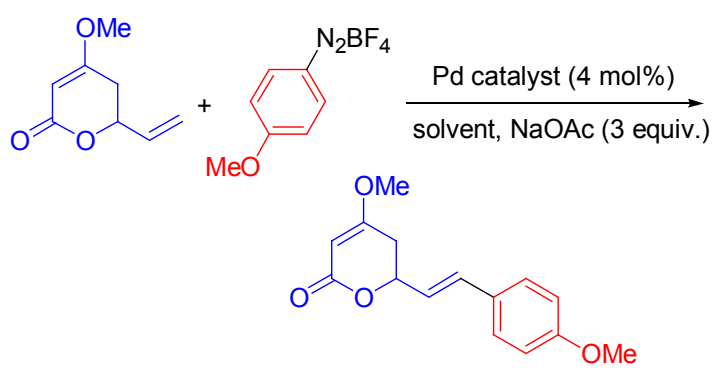

当以 $\mathrm{Pd}_{2}(\mathrm{dba})_{3}$ 为催化剂和 $\mathrm{PhCN}$ 为溶剂时, $80{ }^{\circ} \mathrm{C}$ 下
得到较高产率，并发现微波对反应有促进作用，使反应 的产率达 $85 \%$, 但却使得到的产物无药物活性. 同时发 现当重氮盐连有吸电子基团和游离的羟基时将无法得 到预期产物(Scheme 18).

Sigman 等 ${ }^{[52]}$ 报道了利用零价 $\mathrm{Pd}$ 催化来简单高效地 控制重氮盐与烯烃的反式选择性. 当反应温度降低时, 虽然时间有所延长但使得催化使用量降低，且苯乙烯反 式产物的选择性提高. 研究发现 $\operatorname{Pd}(0)$ 催化在提高反式 选择性的同时，还可以与许多带有官能团的底物反应， 因而比 $\operatorname{Pd}(\mathrm{II})$ 体系更具优势. 此外此反应迅速，无需添 加碱和氧化剂, 也不需要在高温下反应就能获得较高的 收率(Scheme 19).

Correia 等 ${ }^{[53]}$ 首次报道了利用芳基重氮盐实现分子 内参与的 Heck 反应来制备苯，呋喃和吲哚及其衍生物 (Scheme 20). 同时在 CO 氛围下进行羰基化制备得到 3苯并二氢呋喃乙酸及其衍生物(Eq. 32).

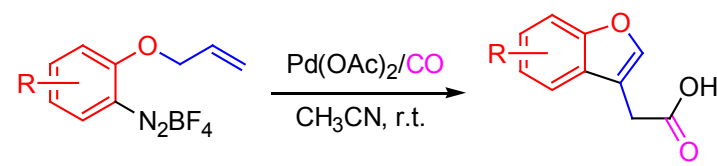

Felpin 等 ${ }^{[54]}$ 报道了以芳基重氮盐参与的 Heck 反应 为关键步骤, 通过钯催化分子内 $\mathrm{C}-\mathrm{H}$ 芳基化和还原环 化反应来合成菲环结构(Scheme 21).

Felpin 等 ${ }^{[55]}$ 报道了芳基重氮盐与 2-芳基丙烯酸酯的 偶联反应. 将 $\mathrm{Pd}(\mathrm{OAc})_{2}$ 溶解到工业级的甲醇中, 从而降 低了催化所需量, 且无需添加额外的碱和配体, 在室温 下即可反应. 反应具有高的反式的区域选择性，并可通 过 DFT 计算予以说明. 将 $\mathrm{Pd}(\mathrm{OAc})_{2}$ 溶解到工业级的甲 醇中，催化简单的 Heck 反应催化最低可至 $0.005 \mathrm{~mol} \%$. 该产物可以经还原环化得到 3-取代的吲哚啉酮衍生物 (Scheme 22).<smiles>[R]OC1=CC(=O)OC(/C=C/c2ccc(OC)cc2)C1</smiles>

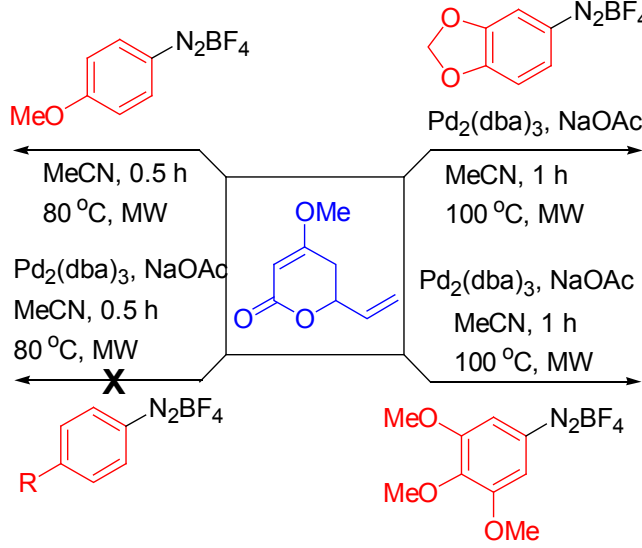

Scheme 18 


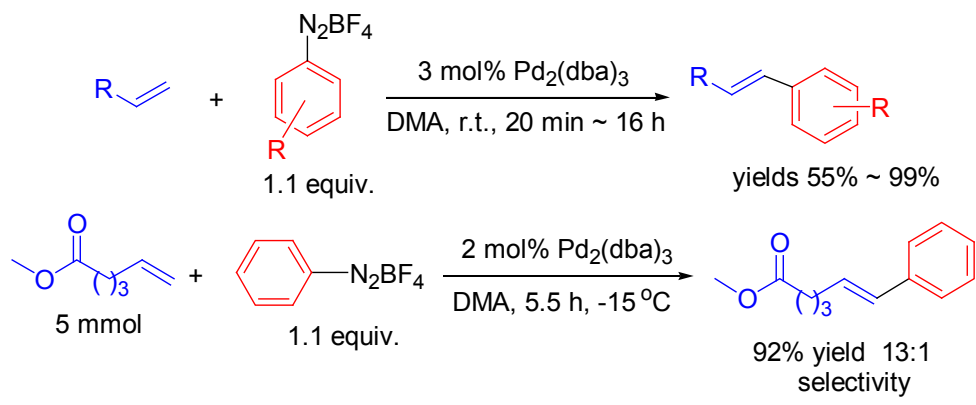

Scheme 19

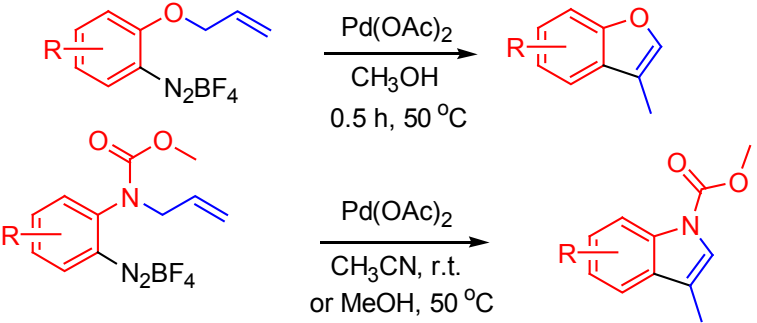

Scheme 20<smiles>[R1]c1ccc(N=[18O])c(Br)c1</smiles>

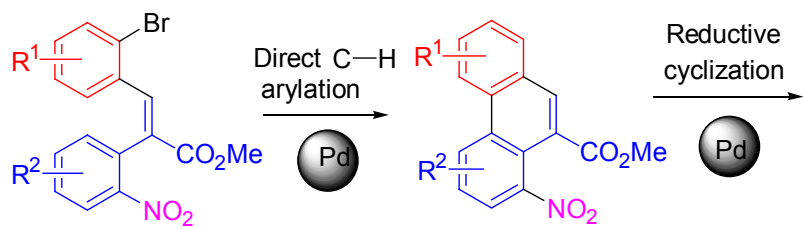<smiles>[R]c1ccc2c3c(cc4ccc[R1]c4c13)C(=O)N2</smiles>

Scheme 21

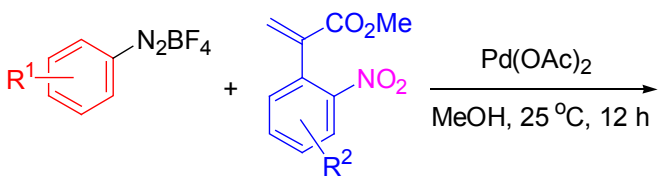

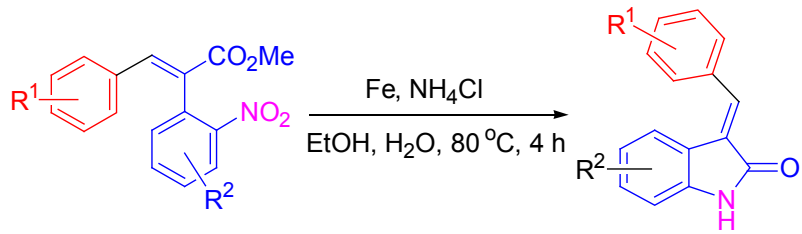

Scheme 22

\section{3 非均相催化的芳基重氮盐参与的偶联反应}

在过去三十多年中, 过渡金属催化的有机反应受到
化学家的广泛关注和研究. 但过渡金属的高昂价格, 催 化剂和反应物的分离困难使合成化学家把更多的目光 转移到可以回收利用并且容易分离的固相负载有机金 属催化剂上. 许多负载型的金属催化剂被设计合成出 来, 并对很多的反应体系起到了不错的催化效果, 有些 已经具有了工业应用的价值 ${ }^{[56]}$. 在均相催化反应中, 催 化剂难以回收和重复使用. 此外, 在高的反应温度下, 可溶性的金属络合物常常因为生成无活性的金属粒子 而失去活性，而多相催化体系则可以克服均相催化体系 以上的缺陷 ${ }^{[57]}$.

Mastrorilli 等 ${ }^{[58]}$ 早在 2005 年就报道了芳基氟硼酸钾 同芳基重氮盐在离子液负载的钯催化下的 Suzuki 类型 的偶联反应(Eq. 33).
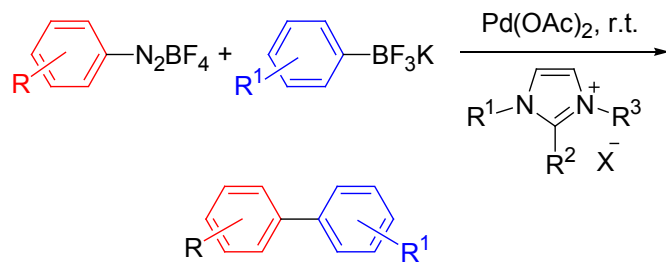

Roglans 等 ${ }^{[59]}$ 报道了芳基氟硼酸钾代替苯硣酸参与 的 Suzuki 反应，利用可循环利用的钯催化剂，以中等产 率得到联苯化合物(Eq. 34).

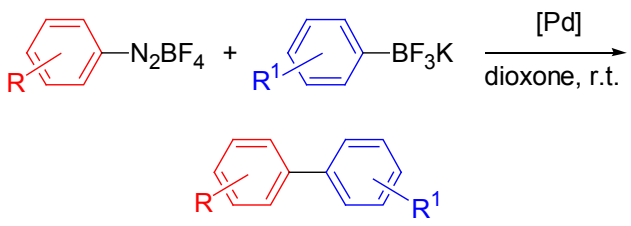

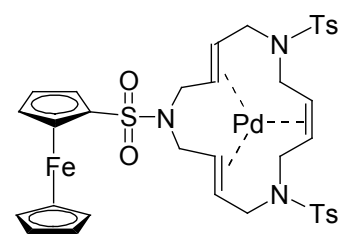

Felpin 等 ${ }^{[60]}$ 报道了在碳负载的零价 $\mathrm{Pd}$ 催化下芳基 重氮盐同苯硼酸的交叉偶联反应，以低毒的醇为溶剂， 反应条件温和、时间短、对环境友好。同时利用芳基重 
氮盐与溴苯的活性差异通过两步反应制备了连三苯, 进 一步说明了芳基重氮盐的高活性(Scheme 23).

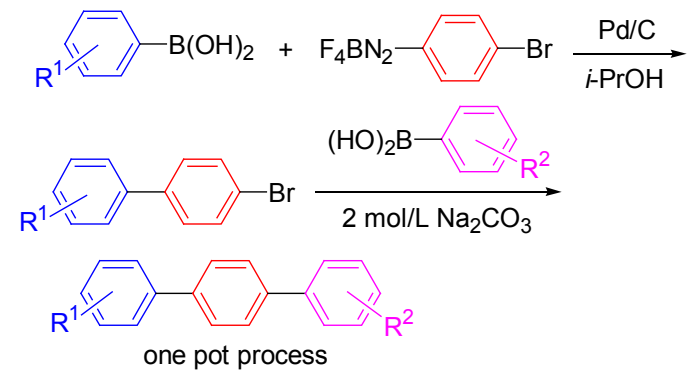

Scheme 23

Luo 等 ${ }^{[61]}$ 报道了利用聚苯乙烯负载的 NHC-Pd 作为 Suzuki-Miyaura 偶联反应的催化剂, 具有高稳定性和可 回收的特点, 经过十次催化循环还能保持高的催化活 性. 使用该催化剂, 反应条件温和无须惰性气体保护, 操作方便，反应产率高(Scheme 24).

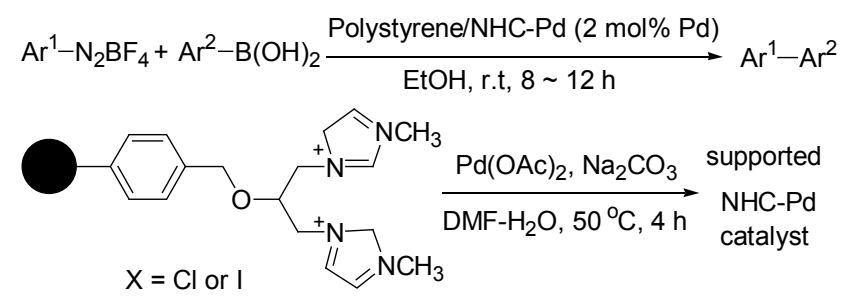

Scheme 24

Felpin 等 ${ }^{[62]}$ 报道了负载在碳酸钡上的零价钯高效和 高选择性的催化了氟嗍酸重氮盐与苯嗍酸的偶联反应, 催化用量能降到 $1 \mathrm{~mol} \%$, 且溶剂为工业级的甲醇, 价格 低廉，反应条件温和且无需加入额外的碱和配体. 同时 能以此为关键步骤合成了联苯肼酯的中间体(Eq. 35).

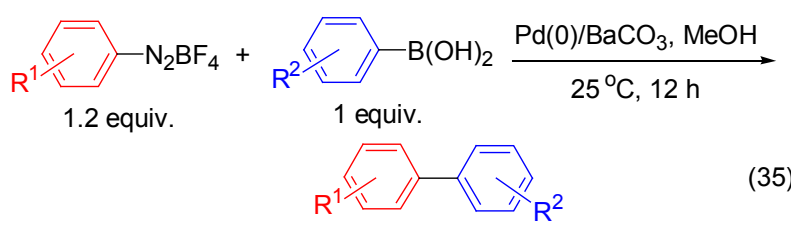

Felpin 等 ${ }^{[63]}$ 报道了以低负载量的钯碳纳米颗粒为催 化剂催化了苯硼酸与四氟苯酸重氮盐的偶联反应, 虽无 法循环利用但有利于产物的分离, 简单高效, 且反应的 范围大, 在室温下反应, 条件温和, 反应无需碱和配体, 以工业级的甲醇为溶剂，绿色环保，反应产率 $62 \%$ 97\%不等(Eq. 36).

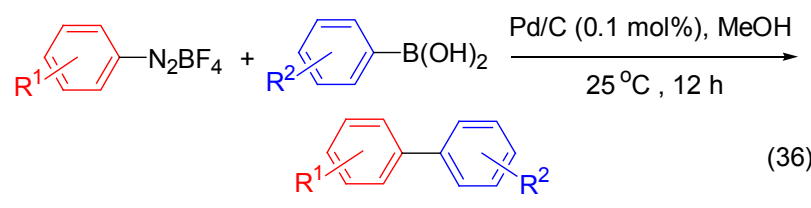

Gras 等 ${ }^{[64]}$ 首次报道了芳基重氮盐与苯硼酸酯的偶 联反应，利用苯硣酸酯的自活化作用，反应过程中无需 添加碱, 且反应条件温和, 对环境友好(Eq. 37).

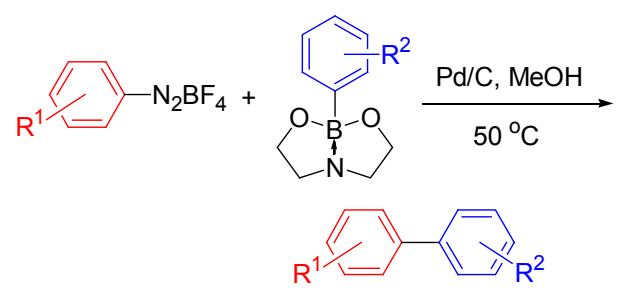

Cacchi 等 ${ }^{[65]}$ 在有氧的条件下, 利用藻酸盐/胶凝糖 天然所形成的圆球材料负载 $0.01 \mathrm{~mol} \%$ 含量的钯催化了 芳基氟硣酸钾与芳基重氮盐的偶联反应，反应以水为溶 剂，反应条件温和，并且产率较高，产率 40\% 90\%不 等, 催化剂可重复利用, 绿色环保(Scheme 25).

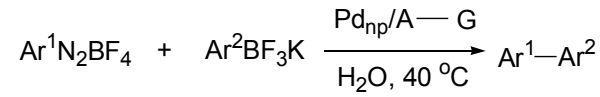

$$
\begin{aligned}
& \mathrm{CaCl}_{2}, \mathrm{H}_{2} \mathrm{O}
\end{aligned}
$$

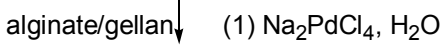

$$
\begin{aligned}
& \text { (2) dehydration and reduction } \\
& \text { alginate/gellan beads } \longrightarrow \mathrm{Pd}_{\mathrm{np}} / \mathrm{A}-\mathrm{G}
\end{aligned}
$$

\section{Scheme 25}

Felpin 等 ${ }^{[66]}$ 报道了利用钯碳负载催化的四氟苯嘲酸 重氮盐与丙烯酸酯的 Heck 反应, 将 Pd 的负载量降至 1 $\mathrm{mol} \%$, 在室温下以工业级的甲醇为溶剂, 得到较高的 产率, 对环境友好, 不足之处是催化剂重复利用性较差 (Eq. 38).

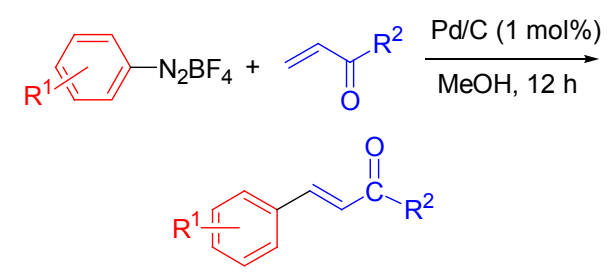

Pore 等 ${ }^{[67]}$ 报道了利用钯纳米颗粒催化的芳基重氮 盐与丙烯酸酯的偶联反应，反应进行时无需添加额外的 碱和配体，也无需氮气保护室温下即可反应，同时以水 为溶剂，反应时间较短，绿色环保且简化了实验操作， 反应具有高产率 $(90 \%$ 及以上)和高区域选择性的特点. 通过加入表面活性剂的 Triton X-100 促使钯分散成纳米 级颗粒而增加催化活性(Eq. 39).

Brunner 等 ${ }^{[68]}$ 报道了通过芳基重氮盐参与的 Heck-Matsuda 反应来制备官能团化的亚苄基丙酮，以碳 酸钙为载体负载钯，室温下就可以高产率合成该类化合 物(Eq. 40). 


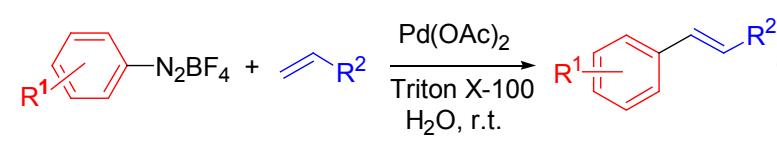

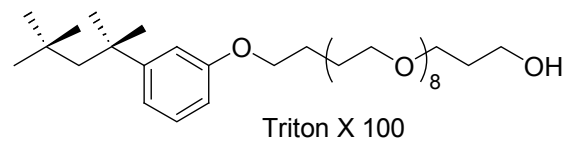

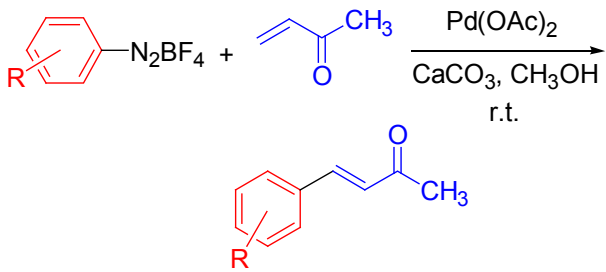

Gholinejad $^{[69]}$ 报道了琼脂糖负载的纳米钯催化 Suzuki-Miyaura 反应, 以甲醇和水为溶剂, 室温下即可 反应，产率 $80 \%$ ～90\%不等. 三次催化循环催化剂的活 性下降不明显(Eq. 41).

$$
\mathrm{R}^{1}-\mathrm{N}_{2} \mathrm{BF}_{4}+\mathrm{R}^{2} \mathrm{~B}(\mathrm{OH})_{2} \underset{\mathrm{MeOH}-\mathrm{H}_{2} \mathrm{O}(5 \mathrm{~mL}), 10 \text { h, r.t. }}{\stackrel{\text { Agaros supported }}{\text { nano } \mathrm{Pd}(0.0026 \mathrm{mmol})}} \mathrm{R}^{1}-\mathrm{R}^{2}
$$

他们还利用琼脂糖负载的纳米钯催化了 Heck- Matsuda 反应, 反应以绿色的水为溶剂, 反应温度温和, 产 率 $80 \% \sim 90 \%$ 不等(Eq. 42).

$$
\mathrm{R}^{1}-\mathrm{N}_{2} \mathrm{BF}_{4}+\mathrm{R}^{2 \curvearrowright} \frac{\begin{array}{c}
\text { Agaros supported } \\
\text { nano } \mathrm{Pd}(0.0026 \mathrm{mmol})
\end{array}}{\mathrm{H}_{2} \mathrm{O}, 3 \mathrm{~h}, 40^{\circ} \mathrm{C}} \mathrm{R}^{1} \curvearrowright \mathrm{R}^{2}
$$

\section{4 绿色体系}

合成化学的理想反应是在 “绿色”、温和的条件下 能以较小的成本得到反应产物, 并且反应物能趋向于百 分之百的选择性和转化率.

Felpin 等 ${ }^{[70]}$ 报道了芳基胺同丙烯酸甲酯的偶联反 应，反应的副产物只有叔丁基醇和氮气，反应中间步骤 产生的芳基重氮盐无需分离. 作者发现间位取代的芳基 胺有利于偶联反应(反应速率快且产率高), 如果要提高 对位取代的芳基胺的产率则只需要添加额外的添加物 (如配体等), 反应无需添加额外的碱, 在室温下即可反 应，并通过理论计算予以说明(Eq. 43).

$$
\mathrm{R}-\mathrm{NH}_{2}+\mathrm{OCH}_{3} \stackrel{\mathrm{Pd}(\mathrm{OAc})_{2}(2.2 \mathrm{~mol} \%)}{\mathrm{MeSO}_{3} \mathrm{H}(20 \mathrm{~mol} \%)}
$$

Barrow 等 ${ }^{[71]}$ 在分段流动的条件下加快了苯胺速率， 从而缩短了反应时间. 对碘苯胺与丙烯酸甲酯得到碘取 代的 Heck 反应产物, 这也说明了重氮盐的活性高于碘 苯(Eq. 44).

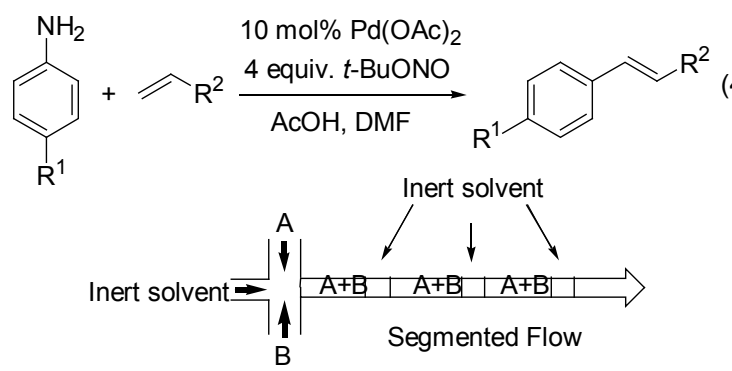

Zarei 等 ${ }^{[72]}$ 报道了硫酸根作为阴离子的芳基重氮盐 在负载在二氧化硅上的钯催化下，与四苯基硼酸钠发生 的非均相偶联反应，不需要加入额外的配体，在室温和 水相中即可反应，并且得到好的产率(Eq. 45).

$$
\mathrm{Ar}^{1} \stackrel{+}{\mathrm{N}_{2}}-\mathrm{OSO}_{3}-\mathrm{SiO}_{2}+\mathrm{NaBPh}_{4} \frac{\mathrm{Pd}(\mathrm{OAc})_{2}(1.5 \mathrm{~mol} \%)}{\mathrm{Na}_{2} \mathrm{CO}_{3}, \mathrm{H}_{2} \mathrm{O} \text {, r.t. }}
$$

Roglans 等 ${ }^{[73]}$ 发展了一种 $\mathrm{PdCl}_{2}\left(\mathrm{CH}_{3} \mathrm{CN}\right)_{2}$ 催化的纯 水作为溶剂的反应，无论是杂环还是苯系芳烃，无论是 Heck 还是 Suzuki 反应都能进行(Scheme 26).

$$
\begin{aligned}
& \mathrm{ArN}_{2} \mathrm{BF}_{4}+\mathrm{HetArBF}_{3} \mathrm{~K} \frac{\mathrm{PdCl}_{2}\left(\mathrm{CH}_{3} \mathrm{CN}\right)_{2}}{\mathrm{H}_{2} \mathrm{O} \text {, r.t. or } 40{ }^{\circ} \mathrm{C}} \mathrm{Ar}-\mathrm{HetAr} \\
& \mathrm{H}_{2} \mathrm{O}, 25^{\circ} \mathrm{C}
\end{aligned}
$$

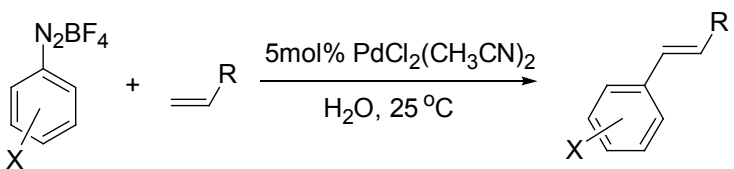

\section{Scheme 26}

最近，我们 ${ }^{[74]}$ 发展了一种纯水作为反应媒介的芳基 重氮盐参与的 Hiyama 和 Suzuki 反应．当芳基硅醚同芳 基重氮盐发生 Hiyama 反应时，由于 $\mathrm{H}_{2} \mathrm{O}$ 分子对 $\mathrm{BF}_{4}$ 离 子的影响无法进攻硅醚中的 $\mathrm{Si}$ 中心原子促进硅醚基团 的离去而不能进行; 但在加入 TBAF 起相转移促进作用 就可以顺利发生; 当芳基氟嗍酸盐同芳基重氮盐发生 Suzuki 反应时, 必须加入碱才能获得较高转化率 (Scheme 27).

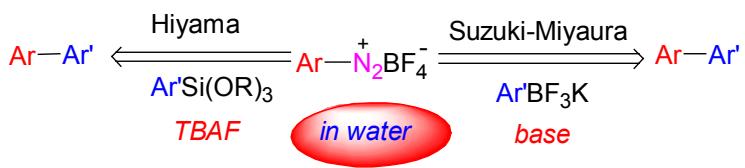

\section{Scheme 27}

我们 ${ }^{[75]}$ 在此基础上又发展了一种钯催化的芳香胺 
通过 “一锅法” 的 Suzuki-Miyaura 反应制备联苯类化合 物的方法. 该反应以水作为溶剂, 无需分离重氮盐中间 体, 直接加入芳基氟硼酸钾、碱和催化剂, 即可较高产 率地得到偶联产物. 反应无需加热和惰性气体保护, 具 有良好的官能团耐受性, 是一种绿色高效的偶联方法. 我们通过热力学计算推测了碱的加入对于反应的促进 作用(Scheme 28).

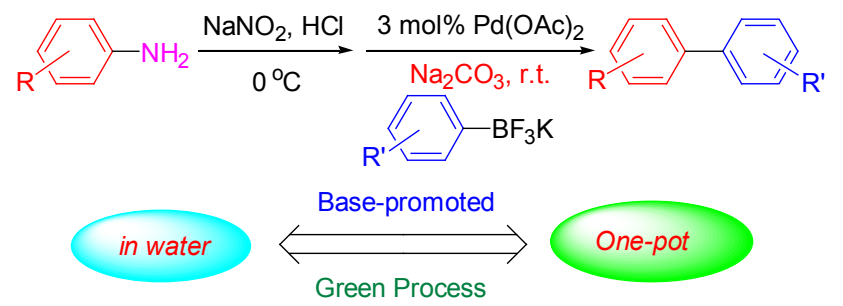

Scheme 28

\section{5 结论与展望}

由于芳基重氮盐参与的偶联反应是近几年才得到 有机化学家的重视, 所以仍然存在有一些问题亟待解 决:

(1)当反应温度较高，时间较长时芳基重氮盐具有 不稳定性, 反应常常伴随有少量副产物生成, 给提纯分 离带来困难.

(2)为了确保均相反应效率需要投入较高剂量的催 化剂，一般在 1 $5 \mathrm{~mol} \%$ ，限制了其在工业上的应用.

(3) 由于多取代芳基重氮盐难以合成，通过芳基重 氮盐进行的 Heck-Matsuda 反应和 Suzuki-Miyaura 反应 一步形成多取代芳烃仍比较困难.

(4)在 Suzuki-Miyaura 反应中, 当邻位有取代基时由 于较拥堵的空间效应而导致转化效率较低, 如何克服位 阻的影响实现高效率的转化依然有待解决.

(5)目前芳基重氮盐的羰基化反应的相关报道相对 较少，而在芳基重氮盐相关的工业应用中有三分之二都 是钯催化的羰基化反应, 所以迫切需要对该类反应进行 深入研究，探索新的有工业应用价值的反应.

(6)目前的 Pd 催化偶联反应的类型主要集中在钯催 化形成 $\mathrm{C}-\mathrm{C}$ 键的反应, 而对于其他的成键反应类型的 研究仍然十分有限, 比如形成 $\mathrm{C}-\mathrm{N}$ 键的 BuchwaldHartwig 氨化反应等.

（7）目前芳基重氮盐反应中应用的过渡金属主要集 中在钯，其他金属的应用十分有限，而廉价金属催化芳 基重氮盐参与的偶联反应的研究从长远来看更加具有 吸引力.

(8)非对映选择性和对映选择性的理论已经比较完 善了. 而芳基重氮盐参与的不对称催化反应仍然是一项 空白. 毫无疑问在未来芳基重氮盐有很广阔的应用前
景，但是仍然有很多问题需要我们解决. 所以我们希望 有针对性的对这些不足之处进行研究，从而使芳基重氮 盐参与的偶联反应更多的应用到实践中来.

\section{References}

[1] Bonin, H.; Fouquet, E.; Felpin, F. X. Adv. Synth. Catal. 2011, 353 3063.

[2] Li, Y. W.; Yan, X. Y.; Li, X.; Chang, H.-H.; Wei, W. L. Shanxi Chem. Ind. 2011, 31, 33.

[3] Taylor, J. G.; Moro, A. V.; Correia, C. R. D. Eur. J. Org. Chem. 2011, 1403.

[4] Xiao, Q.; Zhang, Y.; Wang, J. Acc. Chem. Res. 2013, 46, 236.

[5] Mo, F.; Dong, G.; Zhang, Y.; Wang, J. Org. Biomol. Chem. 2013, 11, 1582 .

[6] Roglans, A.; Pla-Quintana, A.; Moreno-Mañas, M. Chem. Rev. 2006, 106, 4622.

[7] Cheng, K.; Wang, C.; Ding, Y.; Song, Q.; Qi, C.; Zhang, X.-M. J. Org. Chem. 2011, 76, 9261.

[8] Fabrizi, G.; Goggiamani, A.; Sferrazza, A.; Cacchi, S. Angew. Chem., Int. Ed. 2010, 49, 4067.

[9] Wu, X.-F.; Neumann, H.; Beller, M. Chem. Commun. 2011, 47, 7959.

[10] Panda, B.; Sarkar, T. K. Chem. Commun. 2010, 46, 3131.

[11] Wu, X. F.; Schranck, J.; Neumann, H.; Beller, M. Tetrahedron Lett. 2011, 52, 3702.

[12] Wu, X. F.; Neumann, H.; Beller, M. Angew. Chem., Int. Ed. 2011, 50,11142 .

[13] Cacchi, S.; Fabrizi, G.; Goggiamani, A.; Persiani, D. Org. Lett. 2008, 10, 1597.

[14] Cacchi, S.; Fabrizi, G.; Goggiamani, A.; Perboni, A.; Sferrazza, A.; Stabile, P. Org. Lett. 2010, 12, 3279.

[15] Robinson, M. K.; Kochurina, V. S.; Hanna, Jr. J. M. Tetrahedron Lett. 2007, 48, 7687

[16] Cepanec, I.; Litvić, M.; Udiković, J.; Pogorelić, I.; Lovric, M. Tetrahedron 2007, 63, 5614.

[17] Ding, Y.; Cheng, K.; Qi, C.; Song, Q. Tetrahedron Lett. 2012, 53, 6269.

[18] Zhou, J.; Yu, S.; Cheng, K.; Qi, C. J. Chem. Res. 2012, 11, 672.

[19] Vajpayee, V.; Song, Y. H.; Ahn, J. S.; Chi, K. W. Bull. Korean Chem. Soc. 2011, 8, 32.

[20] Zhu, X.; Li, F.; Su, W. Tetrahedron Lett. 2013, 54, 1285.

[21] Xia, Z.; Zhu, Q. Org. Lett. 2013, 15, 4110.

[22] Saini, V.; Liao, L.; Wang, Q.; Jana, R.; Sigman, M. S. Org. Lett. 2013, 15, 5008.

[23] Perez, R.; Veronese, D.; Coelhoband, F.; Antunes, O. Tetrahedron Lett. $2006,47,1325$.

[24] Biajoli, A. F. P.; da Penha, E. T.; Correia, C. R. D. RSC Adv. 2012, 2,11930 .

[25] Severino, E. A.; Costenaro, E. R.; Garcia, A. L. L.; Correia, C. R. D. Org. Lett. $2003,5,305$.

[26] Burtoloso, A. C. B.; Garcia, A. L. L.; Miranda, K. C.; Correia, C. R. D. Synlett 2006, 3145.

[27] Meira, P. R. R.; Moro, A. V.; Correia, C. R. D. Synthesis 2007, 2279.

[28] Garcia, A. L. L.; Carpes, M. J. S.; de Oca, A. C. B. M.; dos Santos, M. A. G.; Santana, C. C.; Correia, C. R. D. J. Org. Chem. 2005, 70, 1050.

[29] Pastre, J. C.; Correia, C. R. D. Org. Lett. 2006, 8,1657.

[30] Silva, K. P.; Godoi, M. N.; Correia, C. R. D. Org. Lett. 2007, 15, 2815. 
[31] Moro, A. V.; Cardoso, F. S. P.; Correia, C. R. D. Tetrahedron Lett. 2008, 49, 5668

[32] Moro, A. V.; dos Santos, M. R.; Correia, C. R. D. Eur. J. Org. Chem. 2011, 7259.

[33] Prediger, P.; Barbosa, L. F.; Génisson, Y.; Correia, C. R. D. J. Org. Chem. 2011, 76, 7737.

[34] de Azambuja, F. D.; Correia, C. R. D. Tetrahedron Lett. 2011, 52, 42.

[35] da Penha, E. T.; Forni, J. A.; Biajoli, A. F. P.; Correia, C. R. D. Tetrahedron Lett. 2011, 52,6342.

[36] Taylor, J. G.; Correia, C. R. D. J. Org. Chem. 2011, 76, 857.

[37] Oliveira, C. C.; dos Santos, E. A. F.; Nunes, J. H. B.; Correia, C. R. D. J. Org. Chem. 2012, 77, 8182.

[38] Barancelli, D. A.; Salles Jr, A. G.; Taylor, J. G.; Correia, C. R. D. Org. Lett. 2012, 14, 6036.

[39] Schwalm, C. S.; Correia, C. R. D. Tetrahedron Lett. 2012, 53, 4836.

[40] Correia, C. R. D.; Oliveira, C. C.; Salles Jr, A. G.; Santos, E. A. F. Tetrahedron Lett. 2012, 53, 3325.

[41] Schwalm, C. S.; Ilton, B. D.; Castro, D.; Ferrari, J.; Fábio, L.; Oliveira, D.; Aparicio, R.; Correia, C. R. D. Tetrahedron Lett. 2012, 53,1660 .

[42] Oliveira, C. C.; Angnes, R. A.; Correia, C. R. D. J. Org. Chem. 2013, 78, 4373

[43] Pastrea, J. C.; Correia, C. R. D. Adv. Synth. Catal. 2009, 351, 1217.

[44] Cacchi, S.; Fabrizi, G.; Goggiamani, A.; Sferrazza, A. Synlett 2009, 1277.

[45] Schmidt, B.; Höter, F.; Berger, R.; Jessel, S. Adv. Synth. Catal. 2010, 352, 2463.

[46] Felpin, F. X.; Miqueu, K.; Sotiropoulos, J. M.; Fouquet, E.; Ibarguren, O.; Laudien, J. Chem. Eur. J. 2010, 16, 5191.

[47] Felpin, F. X.; Barguren, O.; Nassar-Hardy, L.; Fouquet, E. J. Org. Chem. 2009, 74, 1349.

[48] Felpin, F. X.; Coste, J.; Zakri, C.; Fouquet, E.; Chem. Eur. J. 2009, 15,7238 .

[49] Machado, A. H. L.; de Sousa, M. A.; Patto, D. C. S.; Azevedo, L. F. S.; Bombonato, F. I.; Correia, C. R. D. Tetrahedron Lett. 2009, 50, 1222 .

[50] Moro, A. V.; Sega, F.; Cardoso, P.; Correia, C. R. D. Org. Lett. 2009, 16, 3642 .

[51] Soldi, C.; Moro, A. V.; Pizzolatti, M. G.; Correia, C. R. D. Eur. J. Org. Chem. 2012, 3607.
[52] Werner, E. W.; Sigman, M. S. J. Am. Chem. Soc. 2011, 133, 9692.

[53] Siqueira, F. A.; Taylor, J. G.; Correia, C. R. D. Tetrahedron Lett. 2010, 51, 2102.

[54] Nassar-Hardy, L.; Deraedt, C.; Fouquet, E.; Felpin, F. X. Eur. J. Org. Chem. 2011, 4616.

[55] Felpin, F. X.; Miqueu, K.; Sotiropoulos, J. M.; Fouquet, E.; Ibarguren, O.; Laudien, J. Chem. Eur. J. 2010, 16, 5191.

[56] Yan, M.; Feng, X. Chin. J. Org. Chem. 2010, 30, 623.

[57] Yuan, D.; Huang, B. Chin. J. Org. Chem. 2012, 32, 1368.

[58] Gallo, V.; Mastrorilli, P.; Nobile, C.; Paolillo, R.; Taccardi, N. Eur. J. Inorg. Chem. 2005, 582 .

[59] Masllorens, J.; González, I.; Roglans, A. Eur. J. Org. Chem. 2007, 158.

[60] Taylor, R.; Felpin, F. X. Org. Lett. 2007, 15, 2911.

[61] Qin, Y.; Wei, W.; Luo, M. Synlett 2007, 2410.

[62] Felpin, F. X.; Fouquet, E. Adv. Synth. Catal. 2008, 350, 863.

[63] Felpin, F. X.; Fouquet, E.; Zakri, C. Adv. Synth. Catal. 2009, 351, 649.

[64] Bonin, H.; Delbrayelle, D.; Demonchaux, P.; Gras, E. Chem. Commun. 2010, 46, 2677.

[65] Cacchi, S.; Caponetti, E.; Casadei, M. A.; Giulio, A. D.; Fabrizi, G.; Forte, G.; Goggiamani, A.; Moreno, S.; Paolicelli, P.; Petrucci, F.; Prastaro, A.; Saladino, M. L. Green. Chem. 2012, 14, 317.

[66] Felpin, F. X.; Fouquet, E.; Zakri, C. Adv. Synth. Catal. 2008, 350, 2559.

[67] Gaikwad, D.; Pore, D. M. Synlett 2012, 2631.

[68] Stern, T.; Rckbrod, S.; Czekelius, C.; Donner, C.; Brunner, H. Adv. Synth. Catal. 2010, 352, 1983.

[69] Gholinejad, M. Appl. Organomet. Chem. 2013, 27, 19.

[70] Susperregui, N.; Miqueu, K.; Sotiropoulos, J. M.; Callonnec, F.; Fouquet, E.; Felpin, F. X. Chem. Eur. J. 2012, 18, 7210.

[71] Ahmed-Omer, B.; Barrow, D.; Wirth, T. Tetrahedron. Lett. 2009, $50,3352$.

[72] Zarei, A.; Khazdooz, L.; Hajipour, A. R.; Rafiee, F.; Azizi, G.; Abrishami, F. Tetrahedron Lett. 2012, 53, 406.

[73] Bakouri, O. E.; Fernández, M.; Brun, S.; Pla-Quintana, A.; Roglans, A. Tetrahedron 2013, 69, 9761 .

[74] Cheng, K.; Zhao, B.; Hu, S.; Zhang, X.-M.; Qi, C. Tetrahedron Lett. 2013, 54, 6211.

[75] Wei, S.; Wang, C.; Wang, J.; Cheng, K. Chin. J. Org. Chem. 2013, 33,2402 .

(Cheng, F.) 